\title{
Two-level multigrid analysis for the convection-diffusion equation discretized by a discontinuous Galerkin method
}

\author{
M. H. van Raalte and P. W. Hemker ${ }^{*} \dagger$ \\ KdV Institute for Mathematics, University of Amsterdam, Plantage Muidergracht 24, \\ 1018 TV Amsterdam, The Netherlands
}

\begin{abstract}
SUMMARY
In this paper, we study a multigrid (MG) method for the solution of a linear one-dimensional convectiondiffusion equation that is discretized by a discontinuous Galerkin method. In particular we study the convection-dominated case when the perturbation parameter, i.e. the inverse cell-Reynolds-number, is smaller than the finest mesh size.

We show that, if the diffusion term is discretized by the non-symmetric interior penalty method (NIPG) with feasible penalty term, multigrid is sufficient to solve the convection-diffusion or the convection-dominated equation. Then, independent of the mesh-size, simple MG cycles with symmetric Gauss-Seidel smoothing give an error reduction factor of 0.2-0.3 per iteration sweep.

Without penalty term, for the Baumann-Oden (BO) method we find that only a robust (i.e. cellReynolds-number uniform) two-level error-reduction factor $(0.4)$ is found if the point-wise block-Jacobi smoother is used. Copyright (C) 2005 John Wiley \& Sons, Ltd.
\end{abstract}

KEY WORDS: continuous Galerkin method; multigrid iteration; two-level Fourier analysis; point-wise block-relaxation

\section{INTRODUCTION}

The present analysis is motivated by our interest in the hp-self-adaptive solution of elliptic problems that are discretized by discontinuous Galerkin (DG) methods on dyadic grids.

DG methods were traditionally introduced for the solution of hyperbolic equations, as methods that have a natural cell-wise upwind character [1,2]. However, since renewed insights were obtained in their application to elliptic problems, DG methods gain in popularity [3-6], specially because of their convenient properties when combined with the hp-self-adaptive approach and with multigrid (MG) solvers [7-10].

In this framework, having studied the Poisson equation in one and more dimensions $[7,11,12]$, we now study the convergence of the MG method for the one-dimensional

*Correspondence to: P. W. Hemker, CWI, Kruislaan 413, 1098 SJ Amsterdam, The Netherlands.

${ }^{\dagger}$ E-mail: p.w.hemker@cwi.nl 
convection-diffusion equation. In particular we study the convection-dominated case, when the perturbation parameter, i.e. the inverse cell-Reynolds-number, is smaller than the finest mesh size. Although we are even more interested in the more-dimensional convectiondiffusion case, we think that the careful one-dimensional analysis in this paper can be used to obtain some insights into the phenomena encountered and it may tune the expectations for the more-dimensional analysis.

To have a stable DG discretization, we consider for discretization of the diffusion term, the asymmetric DG variants, the Baumann-Oden method and the non-symmetric interior penalty method (NIPG), respectively. For both these methods the discrete operators are positive definite for polynomial discretization of order higher than two [3]. The resulting linear system is block-tridiagonal and, as discussed in Reference [12], can be partitioned in two distinct ways: cell-wise and point-wise. Each partitioning defines its own type of block-relaxation methods that can be used as smoothing procedure in the MG algorithm (e.g. block-Jacobi, block-Gauss-Seidel).

In our analysis we find that, in case of convection-diffusion and the convection-dominated situation and for the DG method where the diffusion term is discretized by the BaumannOden DG method, only a good two-level error-reduction factor $(0.4)$ is predicted if the pointwise block-Jacobi smoother is used. Although with imperfect coarse grid corrections (when the coarse grid problem is solved by approximation) and for vanishing diffusion this blockrelaxation method starts to diverge, the Baumann-Oden-type DG method still can be used in MG techniques when robustness is increased by introducing additional stabilization in the coarse grid correction [13-16].

Further, we show that in case of the DG method, where the diffusion term is discretized by the non-symmetric interior penalty (NIPG) method with feasible penalty term, simple multigrid cycles with cell-wise symmetric Gauss-Seidel are sufficient to solve the convectiondiffusion and the convection-dominated equation. Then an error reduction factor of $0.2-0.3$ per iteration sweep is observed.

For our analysis, we consider the following outline. In Section 2, we give the DG formulation used for the discretization, and we specify the corresponding discrete system. Then, in Section 3, we describe the Fourier analysis for block-Toeplitz operators, where we distinguish between cell-wise and point-wise partitioned stencils. In case the block-Toeplitz operator is partitioned cell-wise, the blocks are associated with the cell-interiors, while in a pointwise partitioned block-Toeplitz operator, the equations and coefficients are associated with the cell-vertices. In the latter partitioning, the coefficients correspond to (vector-valued) grid functions on a regular grid, for which, in view of our two-level analysis, it is natural and easy to define prolongation and restriction operators. This in contrast to cell-wise ordered coefficient, for which we would have to take care of staggered information in coarse and fine cells.

Important for the reliability of the Fourier analysis, is that the inverse of the block-Toeplitz operators are bounded. The consequences of this fact are explained in Section 3.2.

We continue with the smoothing analysis. In Section 4.1, we study the error-amplification operators of the cell- and point-wise block-relaxation methods (block-Jacobi, block-GaussSeidel). To ensure that the equations and coefficients are associated with the cell-vertices, i.e. the coefficients correspond to vector-valued grid functions, both the cell- and point-wise error-amplification operators are casted in point-wise notation.

For mixed convection-diffusion and pure convection, in 4.2, we study the eigenvalue spectra of the error-amplification operators of the cell- and point-wise block-relaxation methods. 
In Section 4.3, we take the MG coarse-grid correction into account and we determine smoothing factors and spectral norms of the block-relaxation methods. In the final section, we show numerical experiments to illustrate the analysis. The present study shows that simple twolevel V-cycles with symmetric Gauss-Seidel smoothing are sufficient to solve the convectiondiffusion equation efficiently, provided that the diffusion term is discretized by the nonsymmetric interior penalty method.

\section{THE DG DISCRETIZATION}

\subsection{The weak formulation for the convection-diffusion equation}

To describe the DG methods considered, we give the variational formulation used for the discretization of the convection-diffusion equation. On the unit cube $\Omega \subset \mathbb{R}^{d}$, we consider the following boundary value problem with Dirichlet and Neumann boundary conditions:

$$
-\varepsilon \Delta u+\mathbf{b} \cdot \nabla u=f \quad \text { on } \Omega, \quad u=u_{0} \quad \text { on } \Gamma_{\mathrm{D}}, \quad \mathbf{n} \cdot \nabla u=g \quad \text { on } \Gamma_{\mathrm{N}}
$$

where $\Gamma_{\mathrm{D}} \cup \Gamma_{\mathrm{N}}=\partial \Omega, \Gamma_{\mathrm{D}} \cap \Gamma_{\mathrm{N}}=\emptyset, \varepsilon \geqslant 0$ and $\mathbf{b} \in \mathbb{R}^{d}$. We further assume that $\mathbf{n} \cdot \mathbf{b} \geqslant 0$ on $\Gamma_{\mathrm{N}}$, i.e. $\Gamma_{\mathrm{N}}$ is an outflow boundary. Next we consider a uniform partitioning of $\Omega$. Therefore, we take a set of regular rectangular cells, $\Omega_{e}$, all of the same size,

$$
\Omega_{h}=\left\{\Omega_{e} \mid \cup_{e} \bar{\Omega}_{e}=\bar{\Omega}, \Omega_{i} \cap \Omega_{j}=\emptyset, i \neq j\right\}
$$

On this partitioning, we define the broken Sobolev space $[4,17]$ of piecewise $H^{1}$-functions, $u$,

$$
H^{1}\left(\Omega_{h}\right)=\left\{u \in L_{2}(\Omega)|u|_{\Omega_{e}} \in H^{1}\left(\Omega_{e}\right), \forall \Omega_{e} \in \Omega_{h}\right\}
$$

Then the DG formulation associated with (1) reads [1,3]: find $u \in H^{1}\left(\Omega_{h}\right)$ such that:

$$
B(u, v)=L(v) \quad \forall v \in H^{1}\left(\Omega_{h}\right)
$$

where

$$
\begin{aligned}
B(u, v)= & \sum_{\Omega_{e} \in \Omega_{h}}(\varepsilon \nabla u, \nabla v)_{\Omega_{e}}-\langle\langle\varepsilon \nabla u\rangle,[v]\rangle_{\Gamma_{\mathrm{int}} \cup \Gamma_{\mathrm{D}}}+\sigma\langle\langle\varepsilon \nabla v\rangle,[u]\rangle_{\Gamma_{\mathrm{int}} \cup \Gamma_{\mathrm{D}}} \\
& +\mu\langle[u], \varepsilon[v]\rangle_{\Gamma_{\mathrm{int}} \cup \Gamma_{\mathrm{D}}} \\
& -\sum_{\Omega_{e} \in \Omega_{h}}(\nabla v, \mathbf{b} u)_{\Omega_{e}}+\left\langle\mathbf{n} \cdot \mathbf{b} u^{-}, v\right\rangle_{\Gamma_{\mathrm{int}}^{-} \cup \Gamma_{\mathrm{D}}}+\langle\mathbf{n} \cdot \mathbf{b} u, v\rangle_{\Gamma_{\mathrm{int}}^{+} \cup \Gamma_{\mathrm{N}}}
\end{aligned}
$$

and

$$
L(v)=\sum_{\Omega_{e} \in \Omega_{h}}(f, v)_{\Omega_{e}}+\sigma\left\langle\langle\varepsilon \nabla v\rangle,\left[u_{0}\right]\right\rangle_{\Gamma_{\mathrm{D}}}+\mu\left\langle\left[u_{0}\right], \varepsilon[v]\right\rangle_{\Gamma_{\mathrm{D}}}+\langle g, \varepsilon v\rangle_{\Gamma_{\mathrm{N}}}
$$

Here $\Gamma_{\text {int }}$ is the union of all interior cell interfaces. With $\Gamma_{\text {int }}^{-}$we denote the set of all inflow boundaries, i.e. $\mathbf{n} \cdot \mathbf{b}<0$, and with $\Gamma_{\text {int }}^{+}$the set of all outflow boundaries. Further, at the interface of two adjacent cells $\Omega_{i}$ and $\Omega_{j},\left.u^{-}\right|_{\Gamma_{\mathrm{int}} \cup \Gamma_{\mathrm{D}}}$ is the upwind value of $u$, obtained from the upwind cell $\Omega_{i}$. Notice that $\left.u^{-}\right|_{\Gamma_{\mathrm{D}}}=u_{0}$, i.e. the value of $u$ at the Dirichlet boundary. The parameters $\sigma$ 
and $\mu$ identify the different DG methods; $\sigma=-1$ for symmetric DG; $\sigma=1$ for the BaumannOden method; $\mu>0$ for, respectively, the symmetric $(\sigma=-1)$ and non-symmetric $(\sigma=1)$ interior penalty DG method (i.e. IPG or NIPG, respectively).

The jump operator $[\cdot]$ and the average operator $\langle\cdot\rangle$ are defined for the traces of functions $v(x)$ and vector functions $\tau(x)$ on the common interface $\Gamma_{i, j}$ between two adjacent $t^{\ddagger}$ cells $\Omega_{i}, \Omega_{j}$. For a scalar function, the operators read

$$
\begin{aligned}
{[v(x)] } & =\left.v(x)\right|_{\partial \Omega_{i}} \mathbf{n}_{i}+\left.v(x)\right|_{\partial \Omega_{j}} \mathbf{n}_{j} \\
\langle v(x)\rangle & =\frac{1}{2}\left(\left.v(x)\right|_{\partial \Omega_{i}}+\left.v(x)\right|_{\partial \Omega_{j}}\right)
\end{aligned}
$$

for $x \in \Gamma_{i, j} \subset \Gamma_{\text {int }} \cup \Gamma_{\mathrm{D}}$. Here $\mathbf{n}_{i}$ is the unit outward pointing normal for cell $\Omega_{i}$. In case of a vector-valued function, $\tau(x)$, we define

$$
\begin{aligned}
& {[\tau(x)]=\left.\tau(x)\right|_{\partial \Omega_{i}} \cdot \mathbf{n}_{i}+\left.\tau(x)\right|_{\partial \Omega_{j}} \cdot \mathbf{n}_{j}} \\
& \langle\tau(x)\rangle=\frac{1}{2}\left(\left.\tau(x)\right|_{\partial \Omega_{i}}+\left.\tau(x)\right|_{\partial \Omega_{j}}\right)
\end{aligned}
$$

To discretize (2), we introduce the finite-dimensional space of piece-wise polynomials of degree at most $k$,

$$
S_{h}=\left\{\phi_{i, e} \in \mathscr{P}^{k}\left(\Omega_{e}\right), i=0,1, \ldots, k, \forall \Omega_{e} \in \Omega_{h}\right\}
$$

Taking test and trial space the same, we consider the discrete equations: find $u_{h} \in S_{h}$ such that

$$
B\left(u_{h}, v_{h}\right)=L\left(v_{h}\right), \quad \forall v_{h} \in S_{h}
$$

\subsection{The discrete system}

To describe the linear system (6), $L_{h} \mathbf{u}_{h}=\mathbf{f}_{h}$, arising from the discrete form (6), we have to specify a base for the space $S_{h}$. For this purpose, on the unit interval we choose the following polynomial basis of degree $2 p-1$ :

$$
\phi_{2 n+k}(t)=t^{n+k}(1-t)^{n+1-k}, \quad n=0,1, \ldots, p-1, \quad k=0,1
$$

On the unit cube, $\hat{\Omega} \subset \mathbb{R}^{d}$, we use a basis of tensor-product polynomials based on (7), and as usual, a basis for $\mathscr{P}^{2 p-1}\left(\Omega_{e}\right)$ is obtained by the affine mapping $\hat{\Omega} \rightarrow \Omega_{e}$.

As we have discussed in Reference [12], a particular advantage of this basis is its hierarchical structure. The first four polynomials represent function values and corrections on derivatives at the cell vertices, while the polynomials of degree $k>3$ are genuine bubble functions and correspond to the interior cell corrections only. So, the corresponding degrees of freedom for these higher order terms can be eliminated by static condensation and, hence, the case $p=2$ is the relevant and generic one for studying iterative solution procedures.

For this initial study, in the present paper we restrict ourselves to the one-dimensional case. In view of the tensor product principle, this is an essential building block for higher-

¥At the Dirichlet boundary, the interface of a flat (virtual) adjacent cell is used. 
dimensional problems. So, taking $p=2$ in (7), writing the approximate solution as

$$
u_{h}=\sum_{e=1}^{N} \sum_{i=0}^{3} c_{i, e} \phi_{i}\left(\frac{x-x_{e}}{h}\right)
$$

the coefficients being determined by the $4 N \times 4 N$ linear system

$$
\begin{aligned}
& \sum_{e=1}^{N} \varepsilon \sum_{i=0}^{3} c_{i, e}\left[\left(\phi_{i, e}^{\prime}(x), \phi_{j, e}^{\prime}(x)\right)_{\Omega_{e}}-\left.\left\langle\phi_{i, e}^{\prime}(x)\right\rangle \cdot\left[\phi_{j, e}(x)\right]\right|_{\Gamma_{\mathrm{int}} \cup \Gamma_{\mathrm{D}}}\right. \\
& \left.+\left.\sigma\left\langle\phi_{j, e}^{\prime}(x)\right\rangle \cdot\left[\phi_{i, e}(x)\right]\right|_{\Gamma_{\mathrm{int}} \cup \Gamma_{\mathrm{D}}}+\left.\mu\left[\phi_{j, e}(x)\right] \cdot\left[\phi_{i, e}(x)\right]\right|_{\Gamma_{\mathrm{int}} \cup \Gamma_{\mathrm{D}}}\right] \\
& \left.\left.+\left.b \sum_{i=0}^{3} c_{i, e}\left[-\left(\phi_{j, e}^{\prime}(x), \phi_{i, e}(x)\right)+\mathrm{n} \phi_{i, e}^{-}(x) \phi_{j, e}(x)\right]\right|_{\Gamma_{\mathrm{int}}^{-}}+\mathrm{n} \phi_{i, e}(x) \phi_{j, e}(x)\right]\left.\right|_{\Gamma_{\mathrm{int}}^{+} \cup \Gamma_{\mathrm{N}}}\right] \\
& \left.\quad=\sum_{e=1}^{N} \sum_{i=0}^{3}\left(f, \phi_{j, e}(x)\right)_{\Omega_{e}}+\left.\sigma\left[u_{0}\right] \cdot\left\langle\varepsilon \phi_{j, e}^{\prime}(x)\right\rangle\right|_{\Gamma_{\mathrm{D}}}+\left.\varepsilon g \phi_{j, e}(x)\right|_{\Gamma_{\mathrm{N}}}-\mathrm{n} b u_{0} \phi_{j, e}(x)\right]\left.\right|_{\Gamma_{\mathrm{D}}}
\end{aligned}
$$

Here, $\mathrm{n}$ is the one-dimensional outward normal vector, i.e. $\mathrm{n}= \pm 1$ and the sign of $b$ determines the convection direction. The resulting matrix is block-tridiagonal and by the constant coefficients (neglecting the influence of the boundary conditions) it can be formulated as a block-Toeplitz matrix, i.e. as a repetition of a lower-diagonal, diagonal and an upper-diagonal $4 \times 4$ block.

This repetition of lower-diagonal, diagonal and an upper-diagonal blocks, can be formulated in two distinct ways. The classical approach is to order the equations and coefficients cell-wise, i.e. $\left\{c_{0, e}, c_{2, e}, c_{3, e}, c_{1, e}\right\}$, then we obtain for the diffusion part of the stencil

$$
L_{h_{\mathrm{D}}} \approx\left[\begin{array}{cccc|cccc|cccc}
-\frac{1}{2} & 0 & -\frac{1}{2} & \frac{1-\sigma}{2}-h \mu & \frac{1+\sigma}{2}+h \mu & \frac{1}{2} & 0 & \frac{-1-\sigma}{2} & \frac{1}{2} \sigma & 0 & 0 & 0 \\
0 & 0 & 0 & \frac{1}{2} \sigma & -\frac{1}{2} \sigma & \frac{2}{15} & \frac{1}{30} & 0 & 0 & 0 & 0 & 0 \\
0 & 0 & 0 & 0 & 0 & \frac{1}{30} & \frac{2}{15} & -\frac{1}{2} \sigma & \frac{1}{2} \sigma & 0 & 0 & 0 \\
0 & 0 & 0 & \frac{1}{2} \sigma & \frac{-1-\sigma}{2} & 0 & \frac{1}{2} & \frac{1+\sigma}{2}+h \mu & \frac{1-\sigma}{2}-h \mu & -\frac{1}{2} & 0 & -\frac{1}{2}
\end{array}\right]
$$

while the stencil for the convection part reads $(b \geqslant 0)$

$$
L_{h_{\mathrm{C}}} \approx\left[\begin{array}{cccc|cccc|cccc}
0 & 0 & 0 & -1 & \frac{1}{2} & \frac{1}{12} & \frac{1}{12} & \frac{1}{2} & 0 & 0 & 0 & 0 \\
0 & 0 & 0 & 0 & -\frac{1}{12} & 0 & \frac{1}{60} & \frac{1}{12} & 0 & 0 & 0 & 0 \\
0 & 0 & 0 & 0 & -\frac{1}{12} & -\frac{1}{60} & 0 & \frac{1}{12} & 0 & 0 & 0 & 0 \\
0 & 0 & 0 & 0 & -\frac{1}{2} & -\frac{1}{12} & -\frac{1}{12} & \frac{1}{2} & 0 & 0 & 0 & 0
\end{array}\right]
$$

So, the discrete block-Toeplitz operator $L_{h}$ for positive convection direction $b$ reads

$$
L_{h} \equiv \frac{\varepsilon}{h} L_{h_{\mathrm{D}}}+L_{h_{\mathrm{C}}}
$$


However, as shown in Reference [12], in view of the Fourier analysis for the multigrid method, where prolongation and restriction operators are based on values at the cell-vertices, it is more convenient to represent the block-Toeplitz operator (11) as a repetition of point-wise partitioned stencils. Then, ordering the equations and coefficients as $\left\{c_{3, e-1}, c_{1, e-1}, c_{0, e}, c_{2, e}\right\}$, we find again that the matrix has a block-Toeplitz structure and we find for the diffusion part of the stencil

$$
L_{h_{\mathrm{D}}} \approx\left[\begin{array}{cccc|cccc|cccc}
0 & 0 & 0 & \frac{1}{30} & \frac{2}{15} & -\frac{1}{2} \sigma & \frac{1}{2} \sigma & 0 & 0 & 0 & 0 & 0 \\
0 & \frac{1}{2} \sigma & \frac{-1-\sigma}{2} & 0 & \frac{1}{2} & \frac{1+\sigma}{2}+h \mu & \frac{1-\sigma}{2}-h \mu & -\frac{1}{2} & 0 & -\frac{1}{2} & 0 & 0 \\
0 & 0 & -\frac{1}{2} & 0 & -\frac{1}{2} & \frac{1-\sigma}{2}-h \mu & \frac{1+\sigma}{2}+h \mu & \frac{1}{2} & 0 & \frac{-1-\sigma}{2} & \frac{1}{2} \sigma & 0 \\
0 & 0 & 0 & 0 & 0 & \frac{1}{2} \sigma & -\frac{1}{2} \sigma & \frac{2}{15} & \frac{1}{30} & 0 & 0 & 0
\end{array}\right]
$$

whereas the stencil for the convection part now reads

$$
L_{h_{\mathrm{C}}} \approx\left[\begin{array}{cccc|cccc|cccc}
0 & 0 & -\frac{1}{12} & -\frac{1}{60} & 0 & \frac{1}{12} & 0 & 0 & 0 & 0 & 0 & 0 \\
0 & 0 & -\frac{1}{2} & -\frac{1}{12} & -\frac{1}{12} & \frac{1}{2} & 0 & 0 & 0 & 0 & 0 & 0 \\
0 & 0 & 0 & 0 & 0 & -1 & \frac{1}{2} & \frac{1}{12} & \frac{1}{12} & \frac{1}{2} & 0 & 0 \\
0 & 0 & 0 & 0 & 0 & 0 & -\frac{1}{12} & 0 & \frac{1}{60} & \frac{1}{12} & 0 & 0
\end{array}\right]
$$

The fact that point-wise ordering leads again to a block-tridiagonal structure is not automatic, but it is caused by the proper choice of basis functions as in (7). The choice makes that the coefficients $\left\{c_{3, e-1}, c_{1, e-1}, c_{0, e}, c_{2, e}\right\}$ correspond to function values and corrections on derivatives at the cell-vertices. Hence, we may interpret the coefficients as four-valued grid functions defined on a regular one-dimensional grid associated with the cell-vertices. These degrees of freedom are also used to define prolongation and restriction operators (see Reference [12]). This, in contrast to the coefficients $\left\{c_{0, e}, c_{2, e}, c_{3, e}, c_{1, e}\right\}$, grouped in the cellwise partitioned stencils, where we would have to deal with staggered information in coarse and fine cells.

We emphasize that, either the repetition of (9) and (10) or the repetition of (12) and (13), represents the same block-Toeplitz matrix $L_{h}$.

\section{FOURIER ANALYSIS FOR BLOCK-TOEPLITZ OPERATORS}

\subsection{Eigenvalues and eigenvectors of a block-Toeplitz operator}

In Reference [12] we derived the Fourier analysis tools to study the smoothing behaviour of the different block-relaxation algorithms. Here, we summarize the results used in this paper. First, we consider an elementary mode $e_{h, \omega}(j h)=\mathrm{e}^{\mathrm{i} j h \omega}$, for all $\omega \in \mathbb{T}_{h} \equiv[-\pi / h, \pi / h]$, defined on the regular infinite one-dimensional grid

$$
\mathbb{Z}_{h}=\{j h \mid j \in \mathbb{Z}, h>0\}
$$



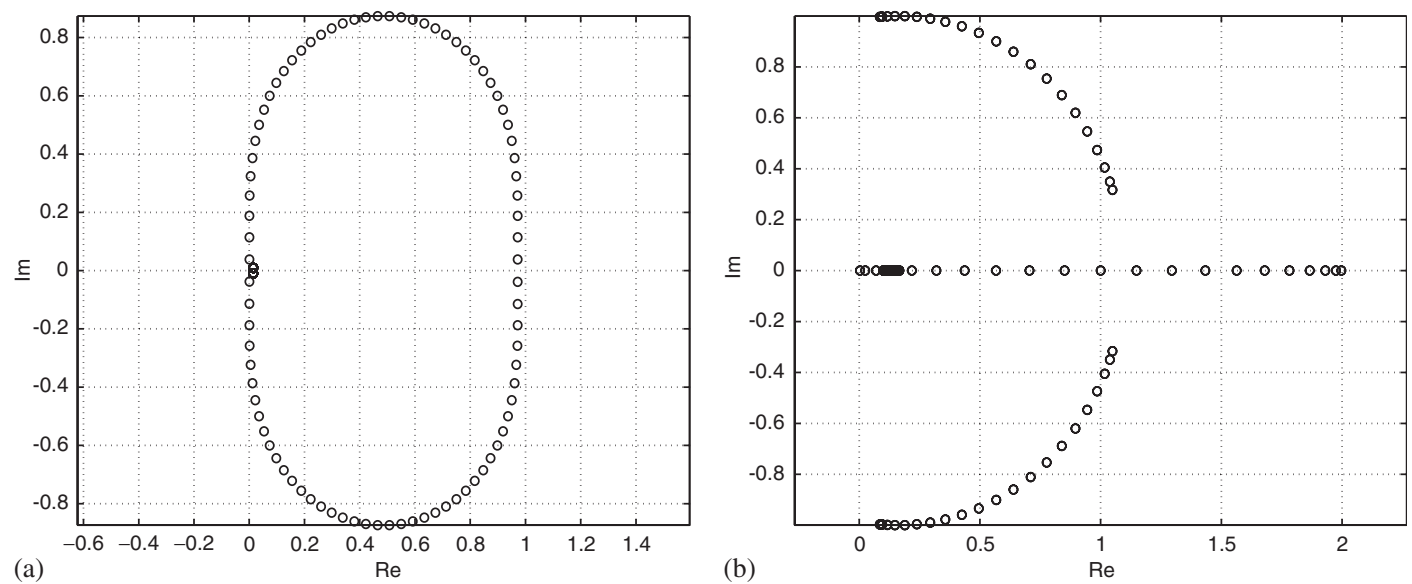

Figure 1. Eigenvalue spectra of the operator $\widehat{L}_{h}(\omega)$, with $\sigma=1$ and $\mu=0$ : (a) convection; (b) diffusion.

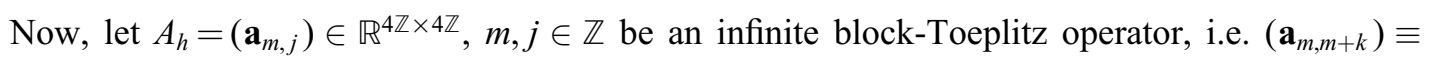
$\left(\mathbf{a}_{-k}\right)$, for $k=j-m \in \mathbb{Z}$ and $\forall m \in \mathbb{Z}$, then we have the identity

$$
\sum_{j \in \mathbb{Z}} \mathbf{a}_{m, j} e_{h, \omega}(j h)=\widehat{A}_{h}(\omega) e_{h, \omega}(m h)
$$

with

$$
\widehat{A}_{h}(\omega)=\sum_{j \in \mathbb{Z}} \mathbf{a}_{m, j} \mathrm{e}^{\mathrm{i}(j-m) h \omega}=\sum_{k \in \mathbb{Z}} \mathbf{a}_{k} \mathrm{e}^{-\mathrm{i} k h \omega}
$$

where $\widehat{A}_{h}(\omega)$ is a $4 \times 4$ matrix, depending on $\omega \in \mathbb{T}_{h}$. If $\widehat{A}_{h}(\omega)$ allows for an eigenvalue decomposition $\widehat{A}_{h}(\omega) V_{h}=V_{h} \Lambda(\omega)$, then with $\left(V_{h} \otimes e_{h, \omega}\right)(j h)=V_{h}(\omega) \mathrm{e}^{\mathrm{i} j h \omega}$, we find that

$$
A_{h}\left(V_{h} \otimes e_{h, \omega}\right)=\left(\sum_{j \in \mathbb{Z}} \mathbf{a}_{m, j} e_{h, \omega}(j h)\right) V_{h}=\widehat{A}(\omega)\left(V_{h} \otimes e_{h, \omega}\right)=\left(V_{h} \otimes e_{h, \omega}\right) \Lambda(\omega)
$$

Then the columns $\mathbf{v}(\omega) e_{h, \omega}(m h)$ of $V_{h} \otimes e_{h, \omega}$ are four-valued grid functions defined on (14) and correspond to the eigenvectors of $A_{h}$, while $\Lambda(\omega)$ is a family of $4 \times 4$ diagonal matrices with the eigenvalues of $A_{h}$ at the diagonal entries.

As an example, we compute the eigenvalue spectra of the discrete Toeplitz operator (11), where, for diffusion stencil (9) (or (12)), we take the Baumann-Oden discretization $(\sigma=1$ and $\mu=0)$. So, recognizing in (11) the different block contributions $\left(\mathbf{a}_{m, j}\right)$, either in cell-wise $((9)$ and $(10))$ or in point-wise $\left((12)\right.$ and (13)) notation, we find $\widehat{L}_{h}(\omega)$ by (15). And because of (16), the four eigenvalues $\lambda_{i}(\omega)$ as function of $\omega \in \mathbb{T}_{h}$ correspond to the eigenvalues of $L_{h}$. Figure 1 shows the eigenvalue spectra for the case of pure convection and pure diffusion.

We see that in both cases the discrete operator is positive semi-definite and hence is also positive semi-definite for mixed convection-diffusion. The zero eigenvalue corresponds to the constant grid function $\mathbf{v}_{0}=\mathbf{v}(\omega) e_{h, 0}(m h)$, given as a column of $V_{h} \otimes e_{h, 0}$. The difference in the 
cell-wise and point-wise partitioning of (11) is reflected in this grid function. Where for the cell-wise partitioned stencils, the constant grid function reads

$$
\mathbf{v}_{0}=\left[\begin{array}{llll}
c & 0 & 0 & c
\end{array}\right]^{\mathrm{T}} e_{h, 0}(m h), \quad c \in \mathbb{R}
$$

for the point-wise partitioned stencils, the constant grid-function is represented by

$$
\mathbf{v}_{0}=\left[\begin{array}{llll}
0 & c & c & 0
\end{array}\right]^{\mathrm{T}} e_{h, 0}(m h), \quad c \in \mathbb{R}
$$

Because the fourth-order discretization (8), both for the Baumann-Oden DG method and for the NIPG method is coercive [3], in our block-relaxation analysis we use these methods for the discretization of the diffusion term.

\subsection{Boundedness of an inverse block-Toeplitz operator}

An important feature in our block-relaxation analysis is that the inverse of a given $n \times n$ block-Toeplitz operator is bounded. We show this by the following simple example.

Let the stencil associated with a $n \times n$ block-Toeplitz operator be given by

$$
A_{h} \cong\left[\begin{array}{lll}
-K & I & \mathbf{0}
\end{array}\right], \quad K \in \mathbb{R}^{n \times n}
$$

Now, writing the inverse of $A_{h}$ as

$$
A_{h}^{-1} \cong\left[\begin{array}{llllllllllll}
\cdot & K^{k} & K^{k-1} & \cdot & K & I & \mathbf{0} & \cdot & \mathbf{0} & \mathbf{0} & \cdot
\end{array}\right]
$$

we readily see that $A_{h}^{-1}$ is bounded if and only if the $n$ eigenvalues $\left|\lambda_{j}(K)\right|<1$. Then, in view of (15), we write

$$
\left(\widehat{A}_{h}(\omega)\right)^{-1}=\frac{I}{I-K \mathrm{e}^{-\mathrm{i} \omega h}}=\sum_{k=0}^{\infty} K^{k} \mathrm{e}^{-\mathrm{i} k h \omega}=\left(\widehat{A}_{h}^{-1}\right)(\omega)
$$

where, using (16), we find the eigenvalues of $A_{h}^{-1}$. However, if $\left|\lambda_{j}(K)\right| \geqslant 1$ the Fourier analysis is not applicable to the finite dimensional case.

\section{SMOOTHING ANALYSIS}

The aim of this study is to identify smoothing procedures which damp the frequencies that cannot be damped by the coarse grid correction in the multigrid process. So, to quantify the smoothing behaviour of simple block-relaxation procedures, we are interested in the spectral radii and spectral norms of the two-level operator for the error. This operator reads

$$
\begin{aligned}
M_{h}^{\mathrm{TLA}} & =\left(M_{h}^{\mathrm{REL}}\right)^{v_{2}} M_{h}^{\mathrm{CGC}}\left(M_{h}^{\mathrm{REL}}\right)^{v_{1}} \\
& =\left(M_{h}^{\mathrm{REL}}\right)^{v_{2}}\left(I-P_{h H} L_{H}^{-1} \bar{R}_{H h} L_{h}\right)\left(M_{h}^{\mathrm{REL}}\right)^{v_{1}}
\end{aligned}
$$

Here, $M_{h}^{\mathrm{REL}}$ is the error-amplification operator of the smoother, $v_{1}$ and $v_{2}$ are the number of pre- (post-) relaxation sweeps, respectively; $M_{h}^{\text {CGC }}$ is the amplification operator of the coarse grid correction (CGC), see e.g. References $[18,19] . P_{h H}$ and $\bar{R}_{H h}$ are the grid transfer operators between the fine $h$-grid and the coarse $H$-grid, where $H=2 h$. 
Because the prolongation should keep the piece-wise cubics invariant, the operator $P_{h H}$ : $S_{H} \rightarrow S_{h}$ is constructed so that $\left(P_{h H} u_{H}\right)(x)=u_{H}(x)$ for all $x \in \mathbb{R} \backslash \mathbb{Z}_{h}$. Its stencil in point-wise ordering is given by

$$
P_{h H} \approx\left[\begin{array}{cccc|cccc|cccc|cccc|cccc}
0 & 0 & 0 & \frac{-1}{8} & 0 & 0 & 0 & \frac{1}{4} & \frac{3}{8} & 0 & 0 & 0 & 0 & 0 & 0 & 0 & 0 & 0 & 0 & 0 \\
0 & 0 & 0 & 0 & 0 & 0 & \frac{1}{2} & \frac{1}{8} & 0 & 1 & 0 & 0 & \frac{1}{8} & \frac{1}{2} & 0 & 0 & 0 & 0 & 0 & 0 \\
0 & 0 & 0 & 0 & 0 & 0 & \frac{1}{2} & \frac{1}{8} & 0 & 0 & 1 & 0 & \frac{1}{8} & \frac{1}{2} & 0 & 0 & 0 & 0 & 0 & 0 \\
0 & 0 & 0 & 0 & 0 & 0 & 0 & 0 & 0 & 0 & 0 & \frac{3}{8} & \frac{1}{4} & 0 & 0 & 0 & \frac{-1}{8} & 0 & 0 & 0
\end{array}\right]
$$

Since test and trial space are the same, the restriction of the residue, $\bar{R}_{H h}$, is the adjoint of the prolongation, $\bar{R}_{H h}=\left(P_{h H}\right)^{\mathrm{T}}$. Notice that this restriction $\bar{R}_{H h}$ for the residue is different from the restriction for the solution $R_{H h}$, which is the left-inverse of $P_{h H}$, defined in Reference [12]. The discrete operator on the coarse grid, $L_{H}$, is the same operator as $L_{h}$, but on the coarse grid. Because of the consistency of the choice of these operators, it is also the Galerkin projection of the fine operator: $L_{H}=\bar{R}_{H h} L_{h} P_{h H}$.

For different $v_{1}$ and $v_{2}$ in (21) the convergence can be studied. However, essential for the coarse grid correction operator for the error, $M_{h}^{\mathrm{CGC}}$, is that it splits the high-frequency modes into a low- and high-frequency mode on the fine grid [20]. Therefore, we better use the smoother before the coarse grid correction (this, in contrast to the correction operator for the residual, for which the smoothing should be applied after the coarse grid correction). So, we restrict ourself to a representative and simple case, where, to quantify the smoothing behaviour of the various block-relaxation methods, we study the spectral radii and spectral norms of $M_{h}^{\mathrm{CGC}} M_{h}^{\mathrm{REL}}$.

In the next section, we specify the block-Jacobi (JOR) and damped block-Gauss-Seidel (DGS) smoothers both for point-wise and for cell-wise relaxation. We further derive Fourier transforms of the error-amplification operators in order to study their eigenvalue spectra. Then we compute the Fourier transforms of the two-level operator $M_{h}^{\mathrm{TLA}}=M_{h}^{\mathrm{CGC}} M_{h}^{\mathrm{REL}}$ to obtain spectral radii and spectral norms, and we quantify the smoothing behaviour of the various block-relaxation methods.

\subsection{Block-relaxation analysis}

A good smoother damps all frequencies in the error that cannot be damped by the coarse grid correction operator $M_{h}^{\mathrm{CGC}}$. Therefore, the spectrum of the amplification operator $M_{h}^{\mathrm{REL}}$ must be such that, at least the eigenvalues corresponding with these frequencies are in absolute value less than one. In this section, we use Fourier analysis to study the amplification operators of both the cell-wise and point-wise block-Jacobi (JOR) and the damped block-Gauss-Seidel (DGS) relaxation methods. In view of the two-level amplification operator $M_{h}^{\mathrm{TLA}}$, studied in Section 4.3, it is of importance that for both the cell-wise and point-wise relaxation procedures the eigenvectors are represented as point-wise grid-functions (i.e. are associated with the cell vertices).

Since we have shown in earlier papers $[7,12]$, that, in the case of pure diffusion, the pointwise smoothers show much better smoothing behaviour than the cell-wise block-relaxation methods, and also because for dominant diffusion $(\varepsilon / h>1$ in (11)), the point-wise smoothers appear to be better in the case when the convection term acts as a small perturbation of the 
Table I. The relaxation methods.

\begin{tabular}{lcc}
\hline & $G_{h}^{-1}$ & $M_{h}^{\mathrm{REL}}=I-G_{h}^{-1} A_{h}$ \\
\hline JOR & $D^{-1}$ & $-D^{-1}(L+U)$ \\
$\mathrm{DGS}_{L}$ & $(D+L)^{-1}$ & $-(D+L)^{-1} U$ \\
$\mathrm{DGS}_{U}$ & $(D+U)^{-1}$ & $-(D+U)^{-1} L$ \\
\hline
\end{tabular}

diffusion, we restrict ourselves here mainly to the case of convection diffusion, $\varepsilon / h \in(0,1]$, and the case of pure convection, $\varepsilon / h=0$.

After the introduction of the Fourier transforms for the various amplification operators in this section, in Section 4.2 we study their eigenvalue spectra for two distinct cases: convectiondiffusion $(\varepsilon / h=1)$ and pure convection $(\varepsilon / h=0)$.

For the DG method with diffusion term discretized by the Baumann-Oden method we will observe that, in case of convection-diffusion, the point-wise amplification operators have eigenvalue spectra with many eigenvalues in absolute value smaller than one, whereas the spectra of the cell-wise amplification operators show eigenvalues in absolute value larger than one. The cell-wise amplification operators can be stabilized if an interior penalty $\mu>0$ is used, i.e. if we consider the NIPG discretization instead of BO discretization for the diffusion term. For pure convection, the cell-wise amplification operators show better smoothing behaviour.

For the discrete system $A_{h} x=b$ we study block-relaxation methods of the type

$$
x^{(k+1)}=x^{(k)}-G_{h}^{-1}\left(A_{h} x^{(k)}-b\right)
$$

where $G_{h}^{-1}$ is an approximate inverse of the matrix $A_{h}$. By decomposing

$$
A_{h}=L+D+U
$$

into a strictly block-lower, a block-diagonal and a strictly block-upper matrix, we easily recognize the block-Jacobi (JOR) and block-Gauss-Seidel (DGS) relaxation methods. The different methods and their amplification operators are shown in Table I.

We first consider decomposition (23) for the point-wise stencils (12) and (13). The stencils corresponding with this decomposition of $A_{h}=\left(\mathbf{a}_{m, j}\right)$ are given in Table II. By (15) the Fourier transforms of the block-diagonal operators read

$$
\widehat{L}(\omega)=\left(\frac{\varepsilon}{h} L_{\mathrm{D}}+L_{\mathrm{C}}\right) \mathrm{e}^{-\mathrm{i} \omega h}, \quad \widehat{D}(\omega)=\left(\frac{\varepsilon}{h} D_{\mathrm{D}}+D_{\mathrm{C}}\right), \quad \widehat{U}(\omega)=\left(\frac{\varepsilon}{h} U_{\mathrm{D}}+U_{\mathrm{C}}\right) \mathrm{e}^{\mathrm{i} \omega h}
$$

So we find the Fourier transform for the amplification operators

$$
\begin{aligned}
\widehat{M_{\mathrm{JOR}}^{\mathrm{REL}}} & =-\widehat{D}^{-1}(\widehat{L}+\widehat{U}) \\
\widehat{M_{\mathrm{DGS}}^{\mathrm{REL}}} & =-(\widehat{D}+\widehat{L})^{-1} \widehat{U} \\
\widehat{M_{\mathrm{DGS}_{U}}^{\mathrm{REL}}} & =-(\widehat{D}+\widehat{U})^{-1} \widehat{L}
\end{aligned}
$$

Decomposing $\widehat{M_{h}^{\mathrm{RE}} \mathrm{L}}(\omega)$ according to (16), we find the eigenvalues $\lambda_{i}(\omega), i=1, \ldots, 4, \omega \in \mathbb{T}_{h}$ of $M_{h}^{\mathrm{REL}}$ and the set of vector-valued eigenfunctions $\mathbf{v}(\omega) e_{h, \omega}(m h)$, defined on the regular infinite one-dimensional grid associated with the cell-vertices. 
Table II. The stencils of the point-wise decomposition (12)-(13).

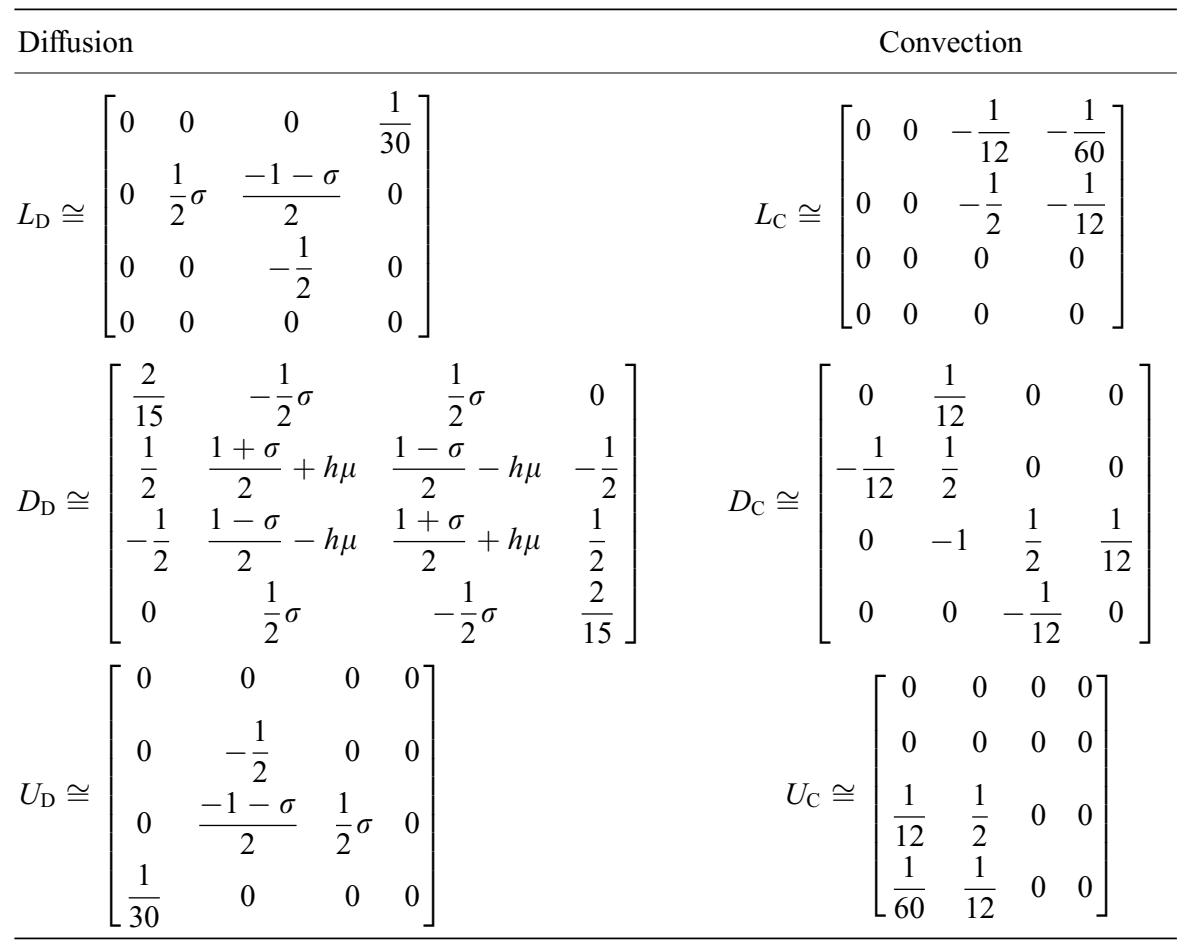

The same procedure can be applied for the cell-wise stencils (9) and (10). However, then the eigenvectors $\mathbf{v} e_{h, \omega}(m h)$ are 4 -valued grid functions associated with the cell-interiors. This is most inconvenient if we want to transfer this information to the coarser grid. Therefore, to also have for the cell-wise amplification operators eigenvectors associated with the cellvertices instead, we rewrite the cell-wise decomposition in point-wise notation, by grouping the equations and coefficients as $\left\{c_{3, e-1}, c_{1, e-1}, c_{0, e}, c_{2, e}\right\}$. The stencils corresponding with this decomposition are shown in Table III, where E denotes the grid-shift operator for which $\widehat{\mathrm{E}}=\mathrm{e}^{\mathrm{i} \omega h}$. Now, with (24) and (25), we find the cell-wise $\widehat{M_{h}^{\mathrm{REL}}}(\omega)$, and the representation is associated with the cell-vertices as needed for the two-level analysis.

\subsection{Eigenvalue spectra of pure smoothers}

Having found the Fourier transforms of the cell- and point-wise block-relaxation operators $M_{h}^{\mathrm{REL}}$ in (25), we can compute their eigenvalue spectra by (16). In order to have the block-relaxation methods independent of the convection direction, we restrict ourselves to Jacobi and symmetric Gauss-Seidel relaxation procedures.

For the discretization of the diffusion term (11), we choose the asymmetric DG methods; the Baumann-Oden $(\sigma=1, \mu=0)$ and the NIPG $(\sigma=1, \mu>0)$ formulation, because these methods, in contrast to the symmetric IPG method, do not suffer from saddle-point behaviour, i.e. the discrete operators do not have eigenvalues with negative real part. 
Table III. The stencils of the cell-wise diagonal decomposition (9)-(10) in point-wise ordering (i.e. grouped as $\left\{c_{3, e-1}, c_{1, e-1}, c_{0, e}, c_{2, e}\right\}$ ).

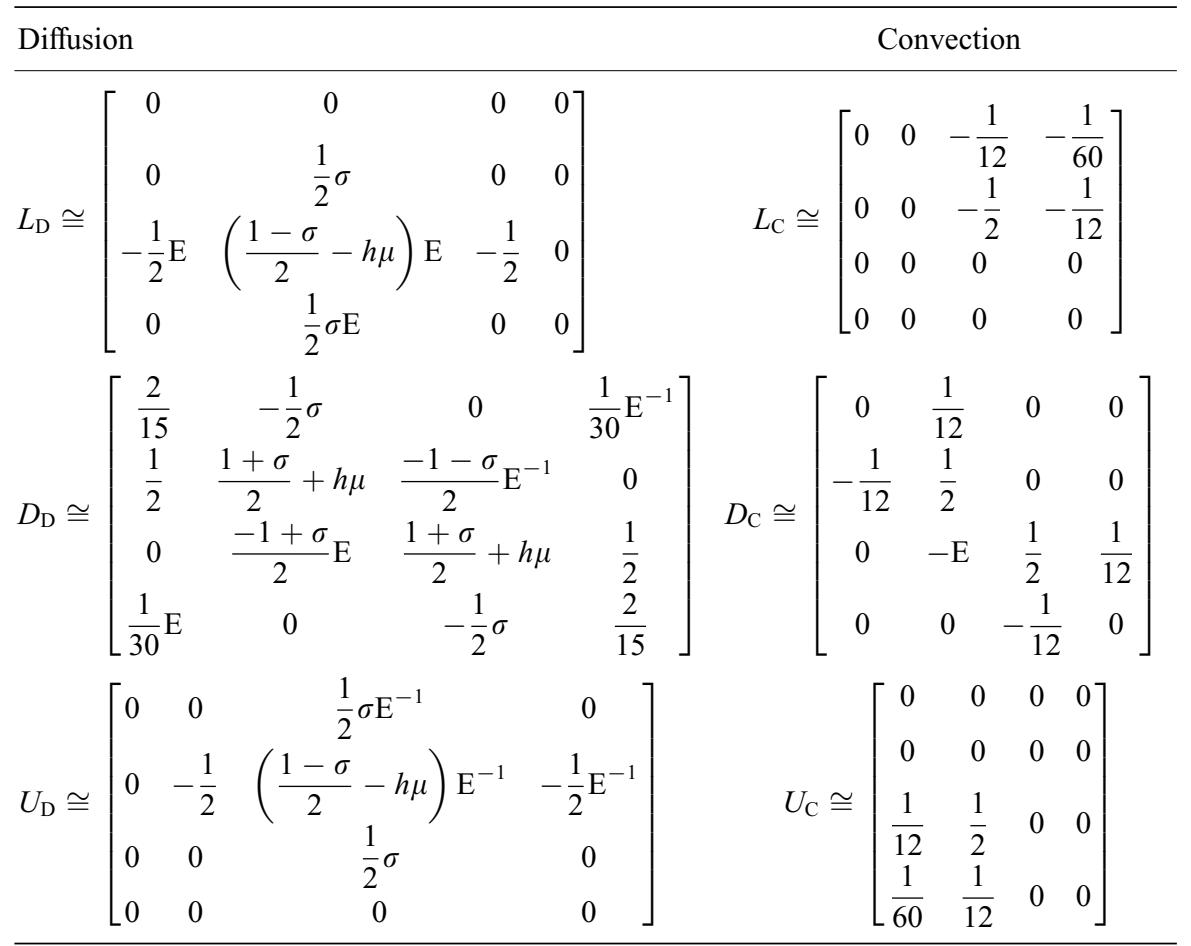

The operator $\mathrm{E}$ denotes a grid-shift so that $\widehat{\mathrm{E}}=\mathrm{e}^{\mathrm{i} \omega h}$.

In Figures 2-7, we show the eigenvalue spectra of Jacobi (JOR) and symmetric GaussSeidel, $M_{\mathrm{SGS}_{U L}}^{\mathrm{REL}}=M_{\mathrm{DGS}_{U}}^{\mathrm{REL}} M_{\mathrm{DGS}_{L}}^{\mathrm{REL}}$, relaxation amplification operators, for DG methods with the diffusion term discretized by the Baumann-Oden DG method and the NIPG method with $\mu=10 / h$ (this, to see the influence of the penalty term on the relaxation).

We first consider the DG method with the diffusion term discretized by the Baumann-Oden DG method (Figures 2 and 3). We see that, similar to the pure diffusion case [12], also for convection-diffusion the point-wise relaxation methods show much better eigenvalue spectra than the cell-wise relaxation methods. Both the eigenvalues corresponding with low-frequency modes (i.e. the frequencies $|\omega|<\pi / 2 h)$ and high-frequency modes $(|\omega| \geqslant \pi / 2 h$ that cannot be represented on the coarse grid), lie within the unit circle in the complex plane. For the point-wise relaxation, the only mode which cannot be damped corresponds to the constant grid function (17). This in contrast to the cell-wise relaxation methods, which may show amplification, especially for the low-frequency modes.

Next we consider the DG method with the diffusion term discretized by the NIPG method. We choose $\mu=10 / h$ to see the influence of the penalty term on the relaxation (Figures 4 and 5). There is a minor influence of the penalty term on the point-wise relaxation operators, but we clearly see a stabilizing effect on the cell-wise relaxation operators.

For the pure convection case (Figures 6 and 7), we see that the cell-wise block-relaxation methods do better than the point-wise relaxation methods. Whereas no amplification occurs in 

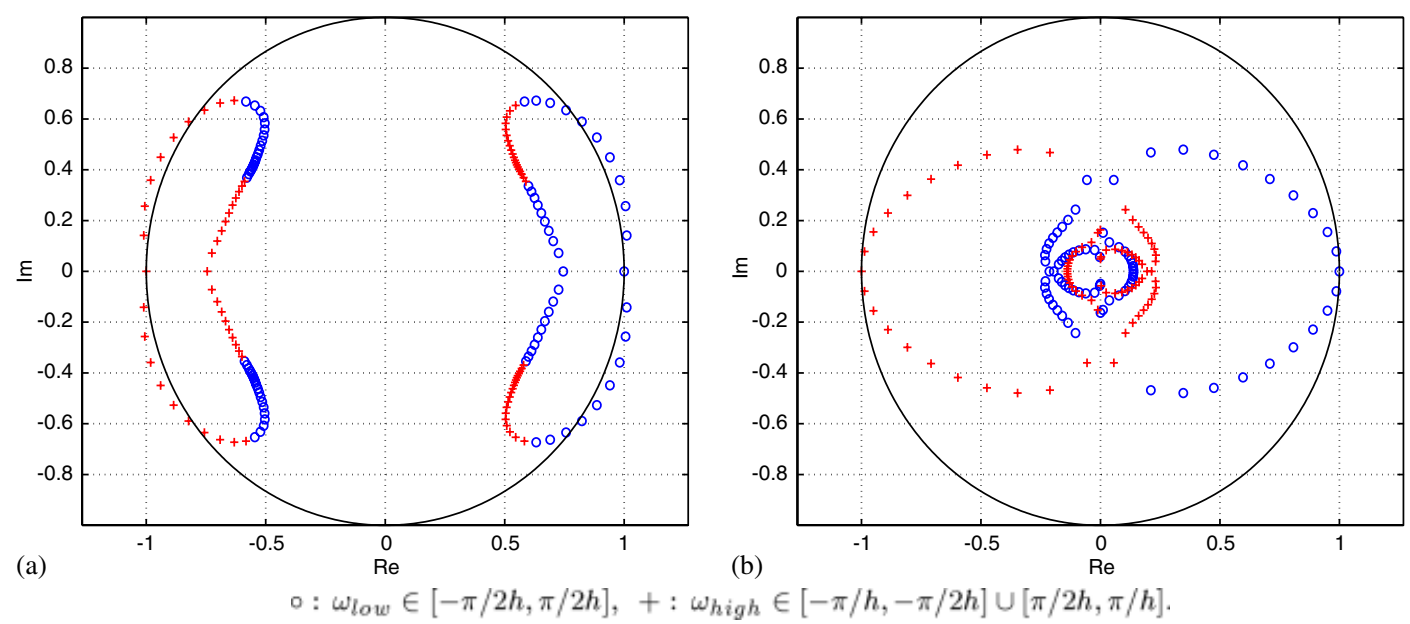

Figure 2. Eigenvalue spectra of $\widehat{M_{\mathrm{JOR}}^{\mathrm{RE}}}(\omega)$, with $\sigma=1, \mu=0$ and $\varepsilon / h=1$ : (a) cell-wise relaxation; (b) point-wise ralaxation.
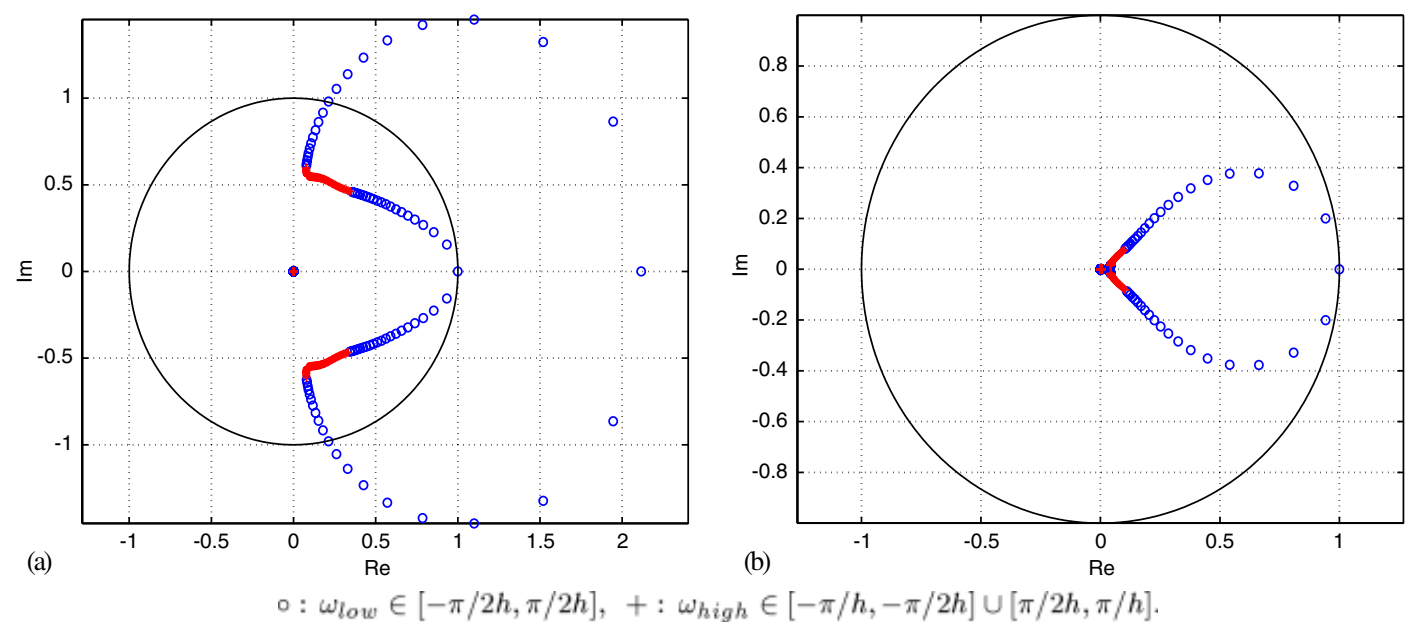

Figure 3. Eigenvalue spectra of $\widehat{M_{\mathrm{SGS}_{U L}}^{\mathrm{REL}}}(\omega)=\widehat{M_{\mathrm{DGS}_{U}}^{\mathrm{REL}}}(\omega) \widehat{M_{\mathrm{DGS}_{L}}^{\mathrm{REL}}}(\omega)$, with $\sigma=1, \mu=0$ and $\varepsilon / h=1$ : (a) cell-wise relaxation; (b) point-wise ralaxation.

case of the Jacobi relaxation, the problem is solved at once by the cell-wise symmetric block Gauss-Seidel relaxation. This is an immediate consequence of the cell-wise upwind character of the DG method. In this situation, the point-wise block-Jacobi diverges (Figure 6). Although all the eigenvalues for the point-wise symmetric block-Gauss-Seidel relaxation (Figure 7) lie within the unit circle (except for the constant mode, which has eigenvalue $\lambda=1$ ), in practice, no convergence is seen. This divergence for the non-periodic, finite-dimensional discrete system is caused by the operator $G_{h}=L+D$. It can be checked that the inverse 

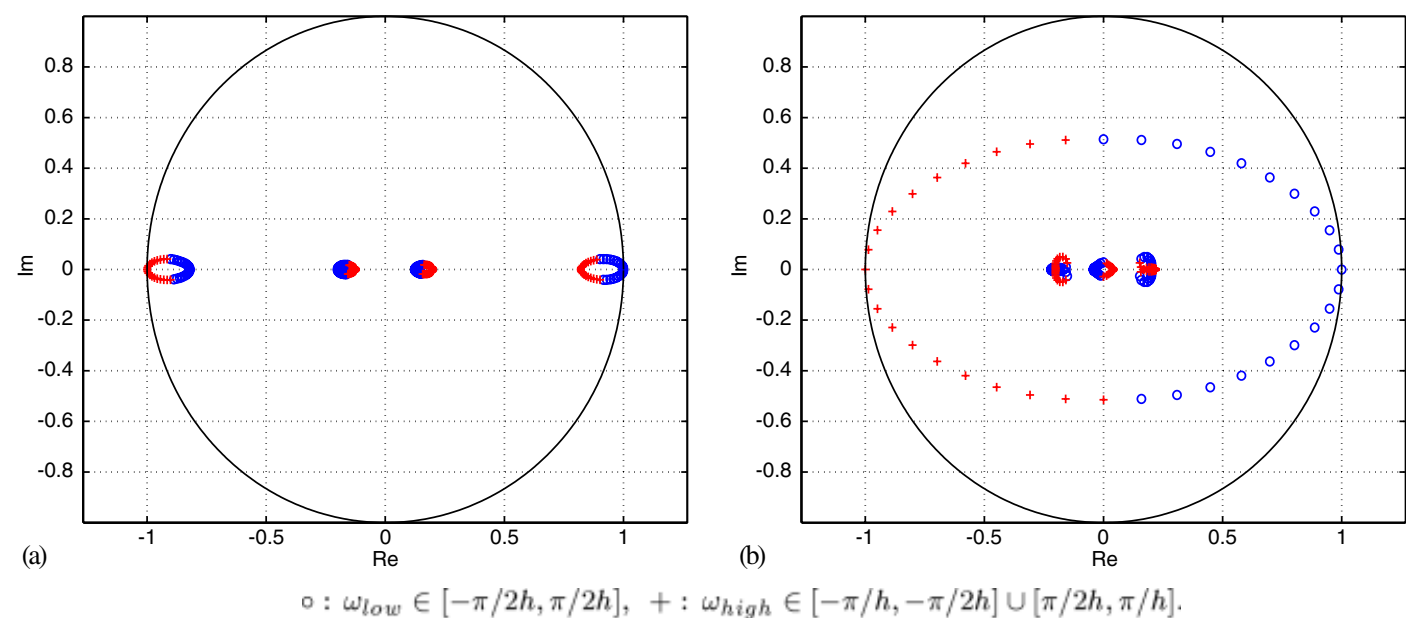

Figure 4. Eigenvalue spectra of $\widehat{M_{\mathrm{JOR}}^{\mathrm{RE}}}(\omega)$, with $\sigma=1, \mu=10 / h$ and $\varepsilon / h=1$ : (a) cell-wise relaxation; (b) point-wise ralaxation.
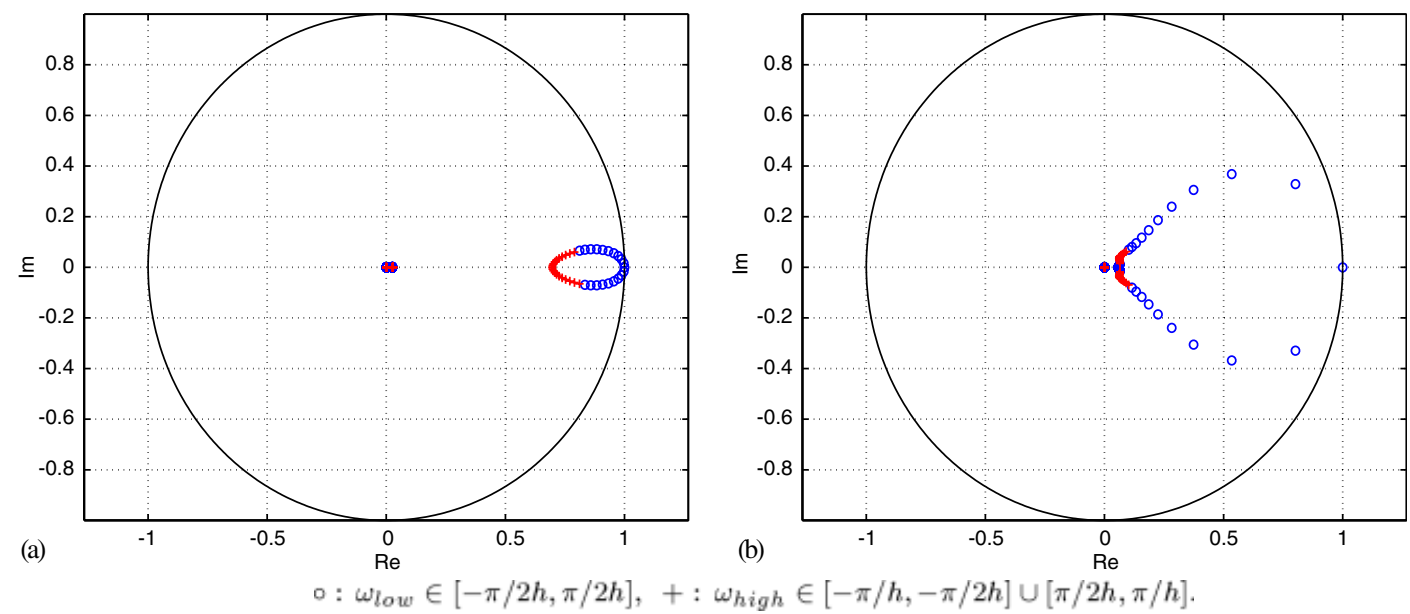

Figure 5. Eigenvalue spectra of $\widehat{M_{\mathrm{SGS}_{U L}}^{\mathrm{REL}}}(\omega)=\widehat{M_{\mathrm{DGS}_{U}}^{\mathrm{REL}}}(\omega) \widehat{M_{\mathrm{DGS}_{L}}^{\mathrm{REL}}}(\omega)$, with $\sigma=1, \mu=10 / h$ and $\varepsilon / h=1$ :

(a) cell-wise relaxation; (b) point-wise ralaxation.

of the corresponding Toeplitz operator is unbounded so that relation (20) is not satisfied. As a consequence, the eigenvalues shown in Figure 3 do not correspond to the convergence behaviour observed.

In the next section, we study the two-level behaviour of the cell- and point-wise blockrelaxation methods. We will observe that, in case of the DG method with diffusion term discretized by the Baumann-Oden, only the point-wise Jacobi relaxation method is a promising smoother, while, in case of the DG method, with diffusion term discretized by the 


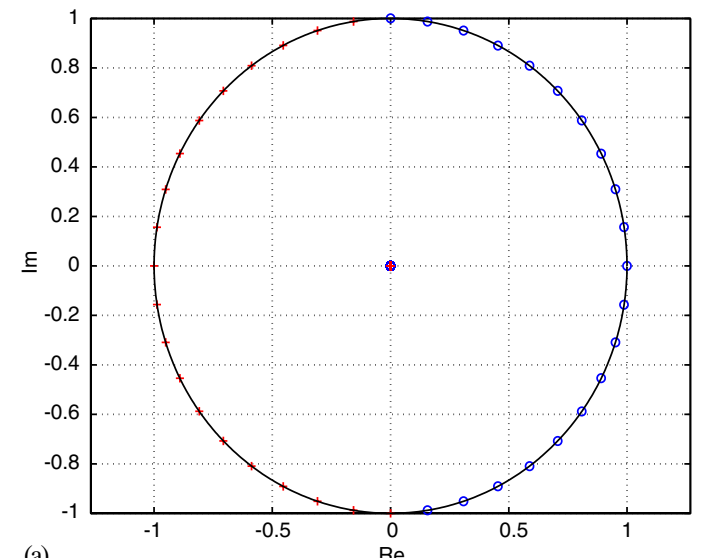

(a)

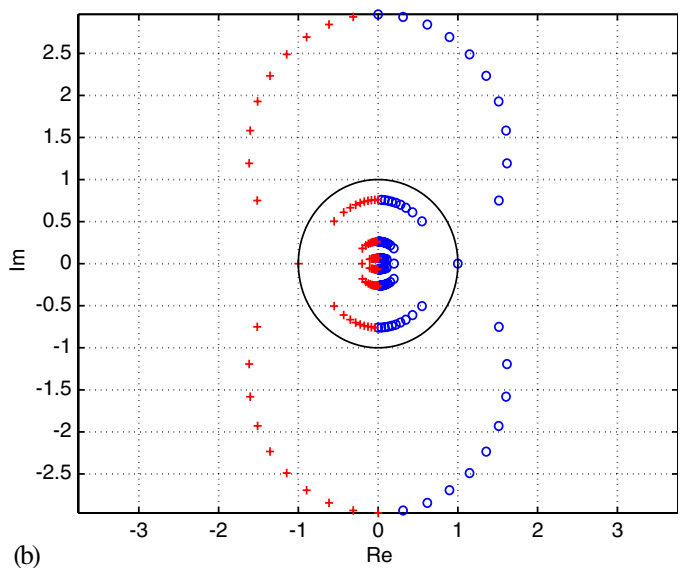

$\circ: \omega_{\text {low }} \in[-\pi / 2 h, \pi / 2 h],+: \omega_{h i g h} \in[-\pi / h,-\pi / 2 h] \cup[\pi / 2 h, \pi / h]$.

Figure 6. Eigenvalue spectra of $\widehat{M_{\mathrm{JOR}}^{\mathrm{RE}}}(\omega)$, with $\varepsilon / h=0$ : (a) cell-wise relaxation; (b) point-wise ralaxation.
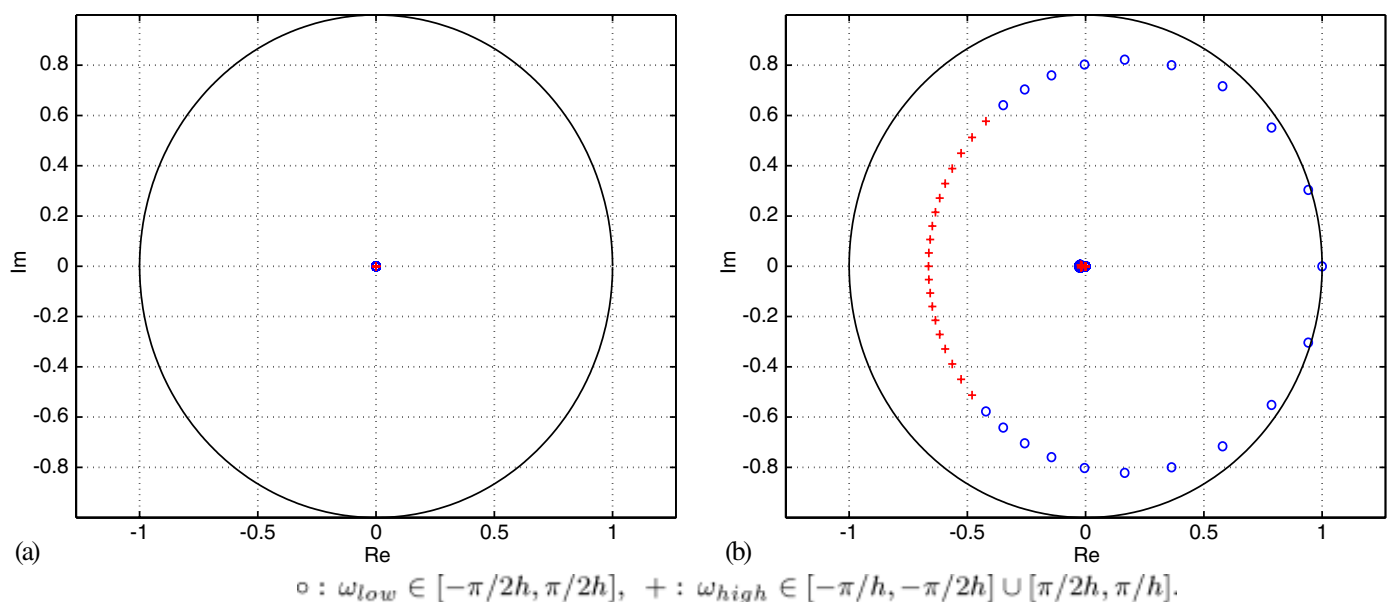

Figure 7. Eigenvalue spectra of $\widehat{M_{\mathrm{SGS}_{U L}}^{\mathrm{REL}}}(\omega)=\widehat{M_{\mathrm{DGS}_{U}}^{\mathrm{REL}}}(\omega) \widehat{M_{\mathrm{DGS}_{L}}^{\mathrm{REL}}}(\omega)$, with $\varepsilon / h=0$ : (a) cell-wise relaxation; (b) point-wise ralaxation.

NIPG method, the cell-wise symmetric Gauss-Seidel relaxation methods show good smoothing behaviour.

\subsection{Two-level analysis}

Having studied the eigenvalue spectra of the various block-relaxation algorithms, we are now interested in the convergence of the two-level operator (21), i.e. the cell- and point-wise JOR and SGS block-relaxation algorithms in combination with the coarse-grid correction. 


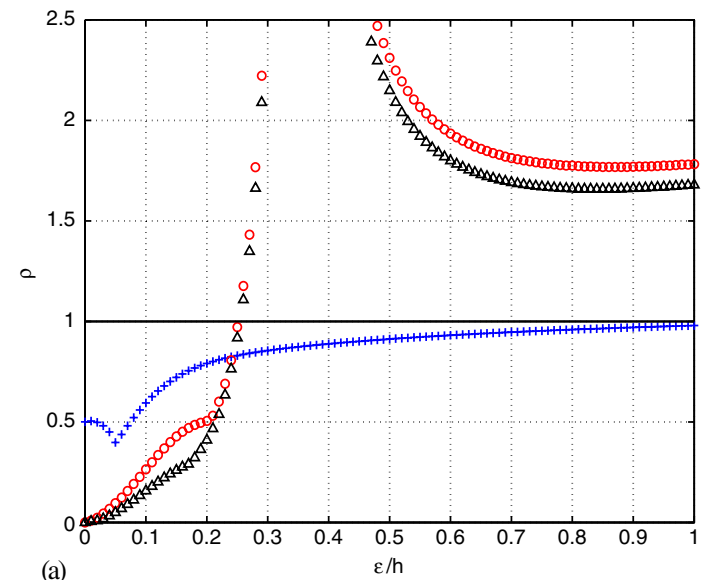

(a)

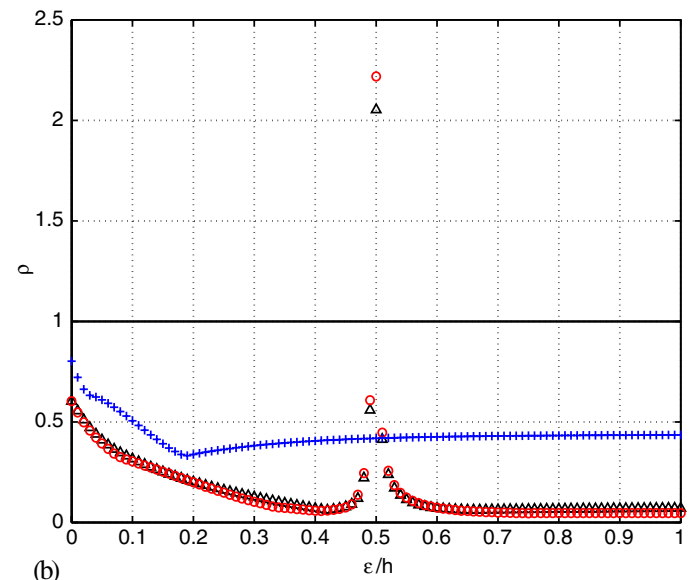

$$
\text { +: JOR; o: } \mathrm{SGS}_{U L} ; \quad \triangle: \mathrm{SGS}_{L U} \text {. }
$$

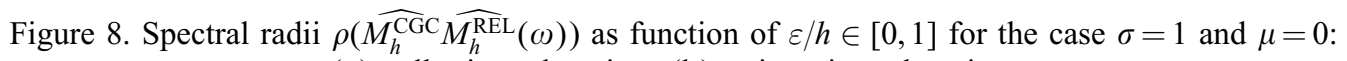
(a) cell-wise relaxation; (b) point-wise ralaxation.

It follows that the Fourier transform of the coarse grid correction for the error $M_{h}^{\mathrm{CGC}}$ reads, with, respectively, $\widehat{P_{h H}}=\widehat{P_{h}}$ and $\widehat{R_{H h}}=\widehat{R_{h}}$, see Reference [12, p. 1027],

$$
\begin{aligned}
\widehat{M_{h}^{\mathrm{CGC}}}(\omega)= & \left(\widehat{I_{h}}-\widehat{P_{h H}} \widehat{L_{H}^{-1}} \widehat{\bar{R}_{H h}} \widehat{L_{h}}\right)(\omega)=\left(\begin{array}{ll}
I & 0 \\
0 & I
\end{array}\right) \\
& \left.\left.-\frac{1}{2}\left(\begin{array}{c}
\widehat{P_{h H}}(\omega) \\
\widehat{P_{h H}}(\omega+\pi / h)
\end{array}\right) \widehat{\left(\bar{L}_{H}\right.}(\omega)\right)^{-1} \widehat{\bar{R}_{H h}}(\omega) \widehat{\bar{R}_{H h}}(\omega+\pi / h)\right)\left(\begin{array}{cc}
\widehat{L}_{h}(\omega) & 0 \\
0 & \widehat{L}_{h}(\omega+\pi / h)
\end{array}\right)
\end{aligned}
$$

for $\omega \in \mathbb{T}_{H}=\mathbb{T}_{2 h} \equiv[-\pi / 2 h, \pi / 2 h]$. Hence, $\widehat{M_{h}^{\mathrm{CGC}}}(\omega)$ is a $8 \times 8$ matrix of which, in view of (16), the eigenvalues $\lambda_{i}(\omega)$ correspond with the eigenvalues of $M_{h}^{\mathrm{CGC}}$.

Interested in the asymptotic convergence of the two-level algorithm, we compute spectral radii of $M_{h}^{\mathrm{CGC}} M_{h}^{\mathrm{REL}}$ for the cell- and point-wise $M_{\mathrm{JOR}}^{\mathrm{REL}}, M_{\mathrm{SGS}_{U L}}^{\mathrm{REL}}=M_{\mathrm{DGS}_{U}}^{\mathrm{REL}} M_{\mathrm{DGS}_{L}}^{\mathrm{REL}}$ and $M_{\mathrm{SGS} L U}^{\mathrm{REL}}=$ $M_{\mathrm{DGS}_{L}}^{\mathrm{REL}} M_{\mathrm{DGS}_{U}}^{\mathrm{REL}}$ block-relaxation methods as function of the diffusion parameter $\varepsilon / h \in[0,1]$. Notice that here $M_{h}^{\mathrm{REL}}(\omega)$ is an $8 \times 8$-matrix and $\omega \in \mathbb{T}_{H}$, which is equivalent with $M_{h}^{\mathrm{REL}}(\omega) \in$ $\mathbb{R}^{4 \times 4}$ and $\omega \in \mathbb{T}_{h}$. We find the spectral radii of $M_{h}^{\mathrm{CGC}} M_{h}^{\mathrm{REL}}$ by computing $\max _{\omega} \rho \widehat{\left(M_{h}^{\mathrm{CGC}}\right.}$ $\widehat{M_{h}^{\mathrm{REL}}}(\omega)$ ), with $M_{h}^{\mathrm{REL}}$ in point-wise notation. The asymptotic convergence factors as function of the diffusion parameter are shown in Figures 8 and 9 for the Baumann-Oden and NIPG method, respectively.

If we consider the convergence for the Baumann-Oden method (Figure 8), we see that the two-level operator with the point-wise Jacobi smoother shows good convergence $(\rho<0.5)$ in the range of $\varepsilon / h>0.1$. Furthermore, this two-level algorithm is stable on the whole interval $\varepsilon / h \in[0, \infty]$. With the cell-wise Jacobi smoother the two-level operator shows good convergence in case of pure convection and convection-dominated problems, $\varepsilon / h \in[0,0.1]$, however, the convergence is very poor in case of convection-diffusion $(\varepsilon / h>0.1)$. 


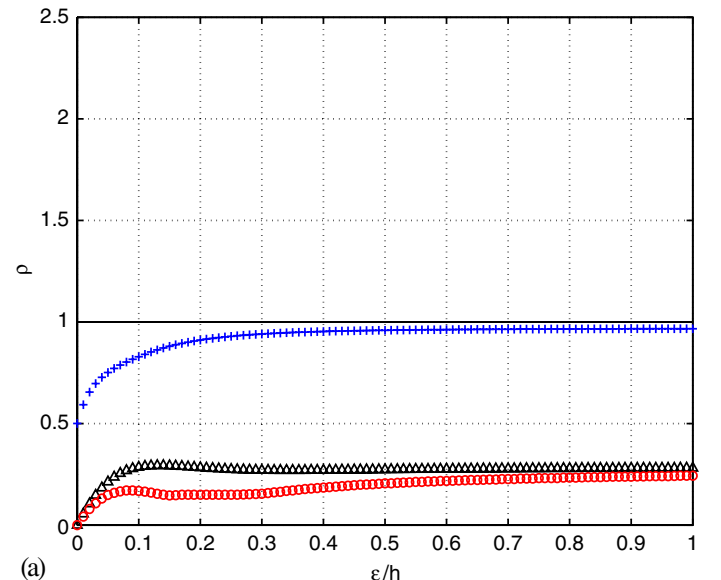

+: JOR; $\quad$ : $\operatorname{SGS}_{U L} ; \quad \triangle: \mathrm{SGS}_{L U}$.

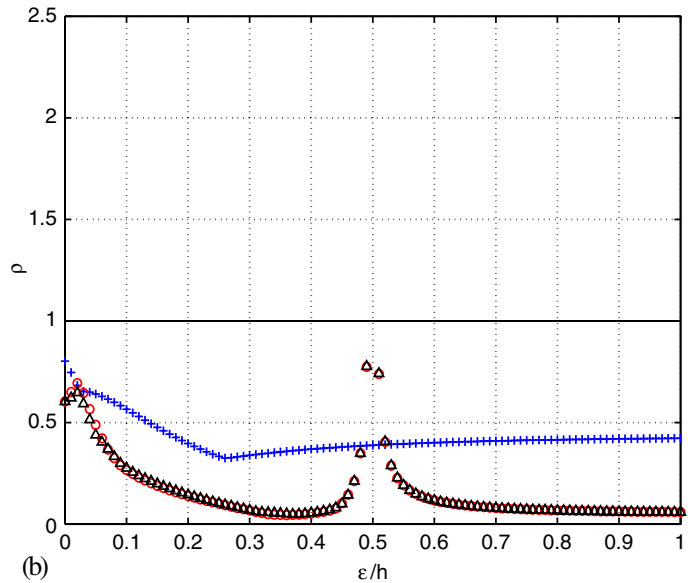

(b)

Figure 9. Spectral radii $\left.\rho \widehat{\left(M_{h}^{\mathrm{CGC}}\right.} \widehat{M_{h}^{\mathrm{REL}}}(\omega)\right)$ as function of $\varepsilon / h \in[0,1]$ for the case $\sigma=1$ and $\mu=10 / h$ : (a) cell-wise relaxation; (b) point-wise ralaxation.

If we consider the symmetric Gauss-Seidel smoothers (Figure 8), in case of the cell-wise block-relaxation methods we see that the spectral radius is generally larger than one and grows without bound in the neighbourhood of $\varepsilon / h \approx 0.35$. We find $\rho<1$ only for $\varepsilon / h<\frac{1}{4}$. In view of this result, we might expect that these two-level algorithms show acceptable convergence for the range of $\varepsilon / h \in\langle 0,0.2]$. (In case of pure convection the problem is solved at once, due to the cell-wise upwind character of the DG method.) However, in practice, the two-level algorithms diverge because relation (20) does not hold for the operator $G_{h}=L+D$ in the down-wind Gauss-Seidel relaxation method (see Section 3.2). Namely, it is easily checked that the inverse of the corresponding Toeplitz operator $G_{h}=L+D$ is unbounded.

A similar situation occurs if we consider the two-level algorithms with the point-wise symmetric Gauss-Seidel smoothers (Figure 8). We may expect good convergence $(\rho<0.1)$ for the range $\varepsilon / h>0.6$. However, in the range $\varepsilon / h \in[0,0.5]$, the finite-dimensional two-level algorithms diverge, again due to the fact that the operator $G_{h}=L+D$ in the down-wind Gauss-Seidel relaxation method has an unbounded inverse.

Now, considering the convergence for the NIPG method in Figure 9, we clearly see the stabilizing effect of the penalty term $(\mu=10 / h)$ on the cell-wise symmetric Gauss-Seidel smoothers. The transition point $(\varepsilon / h \approx 0.35)$, after which the Toeplitz operator $G_{h}=L+D$ has an unbounded inverse, has vanished and relation (20) is restored. So we may expect an overall good convergence $(\rho<0.3)$ for the whole range $\varepsilon / h \in[0, \infty]$. However, the influence of the penalty term on the point-wise block-relaxation algorithms is small: the transition point $\varepsilon / h \approx 0.5$ does not vanish; for smaller values of $\varepsilon / h$ the corresponding finite-dimensional two-level algorithm diverges.

In view of the above analysis, we conclude that for the Baumann-Oden method, if no interior penalty term is used, the point-wise Jacobi algorithm is a good smoother, provided that for $\varepsilon / h \in[0,0.5]$ the problem is solved with sufficient accuracy on the coarser mesh (since the smoother alone diverges). Overall good asymptotic convergence of multigrid cycles 
Table IV. The spectral radii $\rho\left(M_{h}^{\mathrm{CGC}} M_{\mathrm{JOR}}^{\mathrm{REL}}\right)$, for different cases of the diffusion parameter $\varepsilon / h$.

\begin{tabular}{cccccc}
\hline \multicolumn{6}{c}{ Point-wise relaxation } \\
\hline$\varepsilon / h$ & 0 & $1 / 4$ & $1 / 2$ & $3 / 4$ & 1 \\
\hline$\rho\left(M_{h}^{\mathrm{CGC}} M_{\mathrm{JOR}}^{\mathrm{REL}}\right)$ & 0.802 & 0.363 & 0.419 & 0.431 & 0.435 \\
\hline
\end{tabular}

The diffusion term is discretized by the Baumann-Oden DG method.

Table V. The spectral radii $\rho\left(M_{h}^{\mathrm{CGC}} M_{\mathrm{SGS}}^{\mathrm{REL}}\right)$ and $\rho\left(M_{h}^{\mathrm{CGC}} M_{\mathrm{SGS}}^{\mathrm{REL}}\right)$, for different cases of the diffusion parameter $\varepsilon / h$.

\begin{tabular}{cccccc}
\hline \multicolumn{5}{c}{ Cell-wise relaxation } \\
\hline$\varepsilon / h$ & 0 & $1 / 4$ & $1 / 2$ & $3 / 4$ & 1 \\
\hline$\rho\left(M_{h}^{\mathrm{CGC}} M_{\mathrm{SSL}}^{\mathrm{REL}}\right)$ & 0.000 & 0.150 & 0.205 & 0.231 & 0.243 \\
$\rho\left(M_{h}^{\mathrm{CGC}} M_{\mathrm{SGS}}^{\mathrm{RES}_{L U}}\right)$ & 0.000 & 0.274 & 0.273 & 0.279 & 0.281 \\
\hline
\end{tabular}

The diffusion term is discretized by the NIPG method with $\mu=10 / h$.

is found when cell-wise symmetric Gauss-Seidel smoothers are used, and the discretization method is stabilized by the interior penalty term, i.e. if the NIPG discretization is used.

Given the above results, we further restrict ourselves to the more promising methods. The spectral radii, corresponding with Figures 8 and 9, of the point-wise $M_{h}^{\mathrm{CGC}} M_{\mathrm{JOR}}^{\mathrm{REL}}$ and cell-wise $M_{h}^{\mathrm{CGC}} M_{\mathrm{SGS}_{U L}}^{\mathrm{REL}}$ and $M_{h}^{\mathrm{CGC}} M_{\mathrm{SGS}}^{\mathrm{REL}}$ (with $\mu=10 / h$ ) for $\varepsilon / h=0,1 / 4,1 / 2,3 / 4,1$, are shown in the Tables IV and V.

We see that the point-wise two-level algorithm shows acceptable convergence $(\rho \approx 0.4)$ for the various cases of mixed convection-diffusion. However in case of pure convection, the convergence is somewhat poor $(\rho \approx 0.8)$. The cell-wise two-level algorithms show good convergence both for mixed convection-diffusion and for pure convection $(\rho<0.3)$.

Because the spectral radius only predicts the asymptotic rate of convergence, next we check if the methods achieve convergence within a few iteration steps. For this purpose, we compute the 2-norm of the two-level operator. Since both the asymmetric Baumann-Oden and the NIPG formulation are adjoint inconsistent [3], which is reflected in Fourier analysis by unbounded $\left\|\widehat{M_{h}^{\mathrm{CGC}}} \widehat{M_{h}^{\mathrm{REL}}}(\omega)\right\|_{2}$ for vanishing $\omega$, cf. Reference [12], we compute the 2-norm of the two-level operator for the residue,

$$
\bar{M}_{h}^{\mathrm{REL}} \bar{M}_{h}^{\mathrm{CGC}}=L_{h} M_{h}^{\mathrm{REL}} L_{h}^{-1} L_{h} M_{h}^{\mathrm{CGC}} L_{h}^{-1}
$$

The spectral norms of the point-wise unpenalized $\bar{M}_{\mathrm{JOR}}^{\mathrm{REL}} \bar{M}_{h}^{\mathrm{CGC}}$ and the penalized $(\mu=10 / h)$ cell-wise $\bar{M}_{\mathrm{SGS}}^{\mathrm{REL}} \bar{M}_{h}^{\mathrm{CGC}}$ and $\bar{M}_{\mathrm{SGS}}^{\mathrm{REL}} \bar{M}_{h}^{\mathrm{CGC}}$ are shown in Tables VI and VII for the diffusion parameters $\varepsilon / h=0,1 / 4,1 / 2,3 / 4,1$.

We see that, in case of convection-diffusion, for the point-wise Jacobi two-level algorithm the reduction of the residue is guaranteed, from the third iteration step on (Table VI), except for the case of pure convection, where the spectral norm is still large. However, in the next section, we see that this large 2-norm is a conservative upperbound. By the influence of the 
Table VI. The spectral norm $\sigma_{\max }$ after, respectively, one, two and three iterations for the residue, for different cases of the diffusion parameter $\varepsilon / h$.

\begin{tabular}{cccccc}
\hline \multicolumn{5}{c}{ Point-wise relaxation } \\
\hline$\varepsilon / h$ & 0 & $1 / 4$ & $1 / 2$ & $3 / 4$ & 1 \\
\hline$\left\|\bar{M}_{\mathrm{JOR}}^{\mathrm{REL}} \bar{M}_{h}^{\mathrm{CGC}}\right\|$ & 17.656 & 4.748 & 2.600 & 2.040 & 1.829 \\
$\left\|\left(\bar{M}_{\mathrm{JOR}}^{\mathrm{REL}} \bar{M}_{h}^{\mathrm{CGC}}\right)^{2}\right\|$ & 24.788 & 2.265 & 1.131 & 0.887 & 0.797 \\
$\left\|\left(\bar{M}_{\mathrm{JOR}}^{\mathrm{REL}} \bar{M}_{h}^{\mathrm{CGC}}\right)^{3}\right\|$ & 18.671 & 0.569 & 0.443 & 0.374 & 0.343 \\
\hline
\end{tabular}

The diffusion term is discretized by the Baumann-Oden DG method.

Table VII. The spectral norm $\sigma_{\max }$ after, respectively, one and two iterations for the residue, for different cases of the diffusion parameter $\varepsilon / h$.

\begin{tabular}{lccccc}
\hline \multicolumn{5}{c}{ Cell-wise relaxation } \\
\hline \multicolumn{1}{c}{$\varepsilon / h$} & 0 & $1 / 4$ & $1 / 2$ & $3 / 4$ & 1 \\
\hline$\left\|\bar{M}_{\mathrm{SGL}}^{\mathrm{REL}} \bar{M}_{h}^{\mathrm{CGC}}\right\|$ & 0.000 & 1.972 & 1.798 & 1.804 & 1.793 \\
$\left\|\left(\bar{M}_{\mathrm{SGS}}^{\mathrm{REL}} \bar{M}_{h}^{\mathrm{CGC}}\right)^{2}\right\|$ & 0.000 & 0.360 & 0.358 & 0.367 & 0.379 \\
$\left\|\bar{M}_{\mathrm{SGL}}^{\mathrm{REL}} \bar{M}_{h}^{\mathrm{CGC}}\right\|$ & 0.000 & 1.370 & 1.459 & 1.557 & 1.601 \\
$\left\|\left(\bar{M}_{\mathrm{SGS}}^{\mathrm{REL}} \bar{M}_{h}^{\mathrm{CGC}}\right)^{2}\right\|$ & 0.000 & 0.408 & 0.462 & 0.460 & 0.454 \\
\hline
\end{tabular}

The diffusion term is discretized by the NIPG method with $\mu=10 / h$.

boundary condition, in the finite-dimensional case the two-level algorithm already converges within a few iteration steps.

In Table VII, we see for the penalized cell-wise symmetric Gauss-Seidel smoothers that the reduction in the residue is guaranteed after two iteration steps.

\section{NUMERICAL RESULTS}

In this section, we check the theoretical convergence behaviour by numerical experiments, where we restrict ourselves to the more promising two-level algorithms with the unpenalized point-wise Jacobi smoother and the cell-wise penalized symmetric Gauss-Seidel smoothers $(\mu=10 / h)$. For this purpose, we consider the convection-diffusion equation on $[0,1]$,

$$
-\varepsilon u_{x x}+u_{x}=1 \quad \text { with } u(0)=0, \quad u(1)=0, \quad \varepsilon \geqslant 0
$$

which for $\varepsilon \ll 1$ has a sharp boundary layer-type solution, given by

$$
u=x-\frac{\mathrm{e}^{x / \varepsilon}-1}{\mathrm{e}^{1 / \varepsilon}-1}, \quad x \in[0,1]
$$

For the discrete system we use the polynomial basis (7) and we take $h=2^{-7}$. As initial approximation we choose the vector $u_{h}^{0}=[1000, \ldots, \ldots, 1000]$, i.e. a large constant grid 


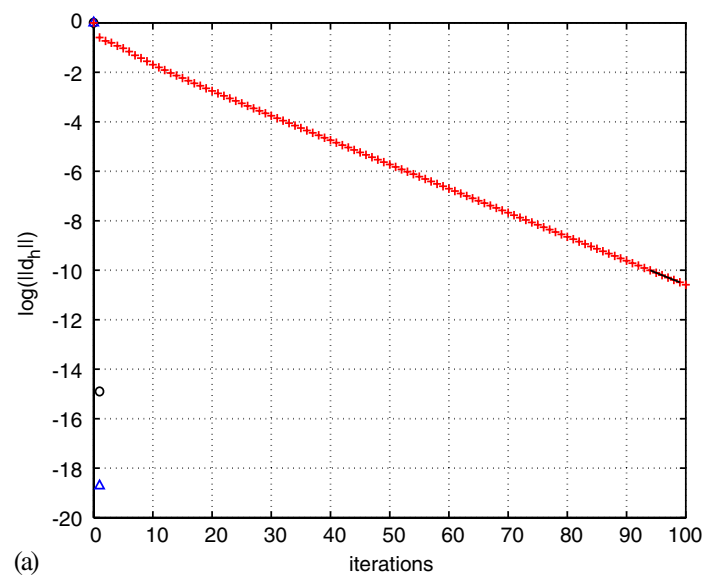

$$
\text { +: JOR; } \triangle: \operatorname{SGS}_{U L} ; \quad \circ: \operatorname{SGS}_{L U} \text {. }
$$

Figure 10. $\log \left(\left\|d_{h}\right\|_{2}\right)$ as function of iterations for the two-level operator on the error with the unpenalized point-wise Jacobi smoother and penalized cell-wise symmetric Gauss-Seidel smoothers $\mu=10 / h$ : (a) $\varepsilon / h=0$; (b) $\varepsilon / h=1$.

function, not satisfying the boundary conditions. For, respectively, the point-wise Jacobi and the cell-wise symmetric Gauss-Seidel relaxation, we apply a single pre-relaxation sweep

$$
u_{h, \mathrm{PRE}}^{i+1}=u_{h, \mathrm{PRE}}^{i}+G_{h}^{-1}\left(f_{h}-L_{h} u_{h, \mathrm{PRE}}^{i}\right)
$$

where $G_{h}^{-1}$ is the approximate inverse of $L_{h}$ as given in Table I. We solve the problem on the coarse grid $H=2^{-6}$, during the coarse grid correction

$$
u_{h}^{i+1}=u_{h, \mathrm{PRE}}^{i+1}+P_{h H} L_{H}^{-1} \bar{R}_{H h}\left(f_{h}-L_{h} u_{h, \mathrm{PRE}}^{i+1}\right)
$$

To be consistent with the Fourier analysis [12], we measure the residue in the 2-norm

$$
\left\|d_{h}\right\|_{2}=\left\|f_{h}-L_{h} u_{h}\right\|_{2}=\sqrt{\sum_{e=1}^{128} \sum_{j=1}^{4} d_{h e, j}^{2}}
$$

The convergence plots for unpenalized point-wise $M_{h}^{\mathrm{CGC}} M_{\mathrm{JOR}}^{\mathrm{REL}}$ and the penalized cell-wise $M_{h}^{\mathrm{CGC}} M_{\mathrm{SGS}_{U L}}^{\mathrm{REL}}$ and $M_{h}^{\mathrm{CGC}} M_{\mathrm{SGS}}^{\mathrm{REL}}$ with diffusion parameters $\varepsilon / h=0,1$ are shown in Figure 10. The convergence factors observed, for diffusion parameters $\varepsilon / h=0,1 / 4,1 / 2,3 / 4,1$ are shown in Tables VIII and IX.

We see that, in case of pure convection, the two-level operator with the point-wise Jacobi smoother converges from the first iteration step, regardless the large 2-norm shown in Table VI. The cell-wise symmetric Gauss-Seidel smoothers solve the problem at once, because of the cell-wise upwind character of the DG method. Also in case of convection-diffusion, convergence is observed from the first iteration step. Furthermore, in all cases of convectiondiffusion, the observed convergence factors coincide very well with the spectral radii shown in Tables IV and V. 
Table VIII. Numerically obtained convergence factors, corresponding with $\rho\left(M_{h}^{\mathrm{CGC}} M_{\mathrm{JOR}}^{\mathrm{REL}}\right)$, for different cases of $\varepsilon / h$.

\begin{tabular}{cccccc}
\hline \multicolumn{6}{c}{ Point-wise relaxation } \\
\hline$\varepsilon / h$ & 0 & $1 / 4$ & $1 / 2$ & $3 / 4$ & 1 \\
\hline$M_{h}^{\mathrm{CGC}} M_{\mathrm{JOR}}^{\mathrm{REL}}$ & 0.80 & 0.36 & 0.42 & 0.43 & 0.44 \\
\hline
\end{tabular}

The diffusion term is discretized by the Baumann-Oden DG method.

Table IX. Numerically obtained convergence factors, corresponding with $\rho\left(M_{h}^{\mathrm{CGC}} M_{\mathrm{SGS} U L}^{\mathrm{REL}}\right)$ and $\rho\left(M_{h}^{\mathrm{CGC}} M_{\mathrm{SGS}}^{\mathrm{REL}}\right)$, for different cases of the diffusion parameter $\varepsilon / h$.

\begin{tabular}{cccccc}
\hline \multicolumn{7}{c}{ Cell-wise relaxation } \\
\hline$\varepsilon / h$ & 0 & $1 / 4$ & $1 / 2$ & $3 / 4$ & 1 \\
\hline$M_{h}^{\mathrm{CGC}} M_{\mathrm{SGS}}^{\mathrm{REL}}$ & 0.000 & 0.14 & 0.20 & 0.23 & 0.25 \\
$M_{h}^{\mathrm{CGC}} M_{\mathrm{SGS}}^{\mathrm{REL}}$ & 0.000 & 0.27 & 0.27 & 0.27 & 0.28 \\
\hline
\end{tabular}

The diffusion term is discretized by the NIPG method with $\mu=10 / h$.

\section{CONCLUSION}

Having shown in earlier papers $[7,12]$ that straightforward multigrid (MG) is quite effective for the solution of the Poisson equation discretized by higher order discontinuous Galerkin (DG) methods, we now study the convergence of MG for the solution of the convectiondiffusion equation.

For the generic fourth-order discretization we consider DG methods with the diffusion term discretized by the asymmetric Baumann-Oden (BO) or the non-symmetric interior penalty method (NIPG) because these methods yield positive definite discrete operators. We study classical multigrid iteration with simple block-relaxation (Jacobi or symmetric Gauss-Seidel) as smoothing procedure. We distinguish two essentially distinct types of block-relaxation algorithms: the classical block-relaxation methods based using a cell-wise partitioning of the discretization matrix, and the new block-relaxation methods based on point-wise partitioning.

By Fourier analysis we show that a robust method, applicable in the whole range of convection-diffusion, dominating convection and pure convection, with acceptable MG convergence, can only be achieved for the Baumann-Oden method if point-wise block-Jacobi smoothing is applied and the problem is solved with sufficient accuracy on the coarser mesh.

However, MG with classical cell-wise symmetric Gauss-Seidel smoothing is quite effective for the NIPG method. Here convergence factors $\rho<0.3$ are found for the whole range of convection-diffusion to pure convection. Moreover, an analysis of the two-level spectral norm shows that an reduction of the residue is guaranteed within two iteration steps.

Thus, the present analysis justifies the use of an interior penalty term in higher order DG methods when MG is applied for the solution of the convection-diffusion equation. 


\section{REFERENCES}

1. Cockburn B. Discontinuous Galerkin methods for convection-dominated problems. In High-Order Methods for Computational Physics, Barth T, Deconink H (eds), Lecture Notes in Computer Science and Engineering, vol. 9. Springer: New York, 1999; 69-224.

2. Johnson C, Pitkäranta J. An analysis of the discontinuous Galerkin method for a scalar hyperbolic equation. Mathematics of Computation 1986; 46:1-26.

3. Arnold DN, Brezzi F, Cockburn B, Marini D. Unified analysis of discontinuous Galerkin methods for elliptic problems. SIAM Journal on Numerical Analysis 2002; 1749-1779.

4. Baumann CE, Oden JT. An $h p$-adaptive discontinuous finite element method for computational fluid dynamics. The University of Texas at Austin, 1997.

5. Nitsche J. Über ein Variationsprinzip zur Lösung von Dirichlet Problemen bei Verwendung von Teilräumen die keinen Randbedingungen unterworfen sind. Abhandlungen aus dem Mathematischen Seminar der Universität Hamburg 1971; 36:9.

6. Prudhomme S, Pascal F, Oden JT, Romkes A. Review of a priori error estimations for discontinuous Galerkin methods. TICAM Report 00-27, 2000.

7. Hemker PW, van Raalte MH. Fourier two-level analysis for higher dimensional discontinuous Galerkin discretisation. Computing and Visualization in Science 2004; 7:159-172.

8. Houston P, Schwab C, Süli E. Discontinuous $h p$-finite element methods for advection-diffusion problems. Technical Report No. 2000-07, ETHZ, Zürich, Switzerland, 2000.

9. Remacle JF, Flaherty JE, Shephard MS. An adaptive discontinuous Galerkin technique with an orthogonal basis applied to compressible flow problems. SIAM Review 2003; 45(1):53-72.

10. Süli E, Schwab C, Houston P. $h p$-DGFEM for partial differential equations with non-negative characteristic form. In Discontinuous Galerkin Methods. Theory, Computation and Applications, Cockburn B, Karniadakis GE, Shu C-W (eds), Lecture Notes in Computer Science and Engineering, vol. 11. Springer: New York, 2000; 221-230.

11. Hemker PW, Hoffmann W, van Raalte MH. Fourier two-level analysis of a multigrid approach for discontinuous Galerkin discretisation with linear elements. Numerical Linear Algebra with Applications 2004; 11:473-491.

12. Hemker PW, Hoffmann W, van Raalte MH. Two-level Fourier analysis of a multigrid approach for discontinuous Galerkin discretisation. SIAM Journal on Scientific Computing 2004; 25:1018-1041.

13. Dahmen W, Müller S, Schlinkmann T. On an adaptive multigrid solver for convection-dominated problems. SIAM Journal on Scientific Computing 2003; 25:1018-1041.

14. de Zeeuw PM, van Asselt EJ. The convergence rate of multi-level algorithms applied to the convection diffusion equation. SIAM Journal on Scientific and Statistical Computing 1985; 6:492-503.

15. Pardhanani A, Spotz W, Carey F. A stable multigrid strategy for convection-diffusion using high order compact discretisation. Electronic Transaction on Numerical Analysis 1997; 6:211-223.

16. Wesseling P. A robust and efficient multigrid method. In Multigrid Methods, Hackbush W, Trottenberg U (eds), Lecture Notes in Mathematics, vol. 960. Springer: New York, 1982; 614-630.

17. Oden JT, Babuška I, Baumann CE. A discontinuous $h p$ finite element method for diffusion problems. Journal of Computational Physics 1998; 146:491-519.

18. Trottenberg U, Oosterlee CW, Schüller A. Multigrid. Academic Press: London, 2001.

19. Wesseling P. An Introduction to Multigrid Methods. Wiley: New York, 1992. Corrected Reprint. R.T. Edwards, Inc.: Philadelphia, 2004.

20. Hemker PW. A note on defect correction processes with an approximate inverse of deficient rank. Applied Mathematics and Computation 1982; 8:137-139. 